\title{
ON FLAG VARIETIES, HYPERPLANE COMPLEMENTS AND SPRINGER REPRESENTATIONS OF WEYL GROUPS
}

\author{
G. I. LEHRER and T. SHOJI \\ (Received 26 April 1989; revised 3 August 1990) \\ Communicated by $\mathrm{H}$. Lausch \\ mathematics in Australia on the occasion of his retirement
}

Dedicated to G. E. (Tim) Wall in recognition of his distinguished contribution to

\begin{abstract}
Let $G$ be a connected reductive linear algebraic group over the complex numbers. For any element $A$ of the Lie algebra of $G$, there is an action of the Weyl group $W$ on the cohomology $H^{i}\left(\mathscr{B}_{A}\right)$ of the subvariety $\mathscr{B}_{A}$ (see below for the definition) of the flag variety of $G$. We study this action and prove an inequality for the multiplicity of the Weyl group representations which occur ((4.8) below). This involves geometric data. This inequality is applied to determine the multiplicity of the reflection representation of $W$ when $A$ is a nilpotent element of "parabolic type". In particular this multiplicity is related to the geometry of the corresponding hyperplane complement.
\end{abstract}

1980 Mathematics subject classification (Amer. Math. Soc.) (1985 Revision): 20 G 40.

\section{Introduction}

Let $G$ be a connected reductive linear algebraic group over the complex number field $\mathbb{C}$, let $B$ be a Borel subgroup of $G$ and $A$ a nilpotent element in the Lie algebra $\mathscr{G}$ of $G$. Consider the subvariety $\mathscr{B}_{A}$ of the flag variety $\mathscr{B}=G / B$ defined by $\mathscr{B}_{A}=\{g B \in \mathscr{B} \mid A \in$ Adg Lie $B\}$.

The research of the first named author was supported in part by an ARGS Grant.

The second named author is grateful to the Department of Pure Mathematics, University of Sydney for its hospitality during his stay in 1988 .

(C) 1990 Australian Mathematical Society $0263-6115 / 90 \$ A 2.00+0.00$ 
It is well-known from the work of Deligne, Lusztig, Springer and Kazhdan that the cohomology theory of the varieties $\mathscr{B}_{A}$ enters the representation theory of reductive groups over finite fields. The connection arises as follows. Think of $G$ as a group scheme over $\mathbb{Z}$, and let $G_{p}$ ( $p$ a prime) be its group $G\left(\overline{\mathbb{F}}_{p}\right)$ of $\overline{\mathbb{F}}_{p}$ points, with a Frobenius endomorphism $F$, corresponding to an $\mathbb{F}_{q}$-structure on $G_{p}$ (where $q$ is a power of $p$ ). Assume for simplicity that $G_{p}$ is $\mathbb{F}_{q}$-split, and write $G^{F}$ for $G_{p}^{F}$.

Deligne and Lusztig [1] have defined virtual $\overline{\mathbb{Q}}_{l}$-modules $R_{T}^{G}(\theta)$ of $G^{F}$ (where $l$ is a prime distinct from $p$ ), where $T$ is an $F$-stable maximal torus of $G$ and $\theta \in\left(T^{F}\right)^{-}$, whose characters are given by a formula which essentially reduces the computation of all character values to that of values on unipotent elements of $G^{F}$.

Take a unipotent element $u \in G^{F}$. Then $R_{T}^{G}(\theta)(u)$ is independent of $\theta$, and we may write $R_{T}^{G}(\theta)(u)=Q_{T}^{G}(u)$. The functions $Q_{T}^{G}(u)$ are called the Green functions of $G^{F}$ and a part of the character theory of $G^{F}$ has been reduced (cf. Lusztig [14]) to their explicit computation.

Now for $p$ and $q$ sufficiently large, the nilpotent classes in $\mathscr{G}=\mathscr{G}_{\mathbb{C}}$ have the same classification as the unipotent classes in $G_{p}$. Further the $G^{F}$-classes into which the $G_{p}$-class of $u \in G^{F}$ splits are classified by $F$-conjugacy classes in $C(u)=Z_{G}(u) / Z_{G}(u)^{0}$ (see, for example, [27]). Write $\left\{u_{c} \mid c \in C(u)\right\}$ for a representative set of elements of the $G^{F}$-classes of $G_{p}$-conjugates of $u$ in $G^{F}$ and let $A \in \mathscr{G}$ be in the nilpotent orbit corresponding to $u$ (see above). It follows from the work of Shoji [18] and Spaltenstein [24] that when $G$ is not of type $E_{8}$, in each $F$-stable $G_{p}$-conjugacy class of unipotent elements, there is an element $u \in G^{F}$ such that $F$ acts trivially on $C(u)$ and on the set of irreducible components of $\mathscr{B}_{u}\left(=\left\{g B \in \mathscr{B} \mid u \in g B g^{-1}\right\}\right)$. These elements are called "split" (or, in [18], "distinguished") and we shall choose notation so that $u$ (above) $=u_{1} \quad(1 \in C(u))$ is split.

The $G^{F}$-conjugacy classes of maximal tori $T$ in $G_{p}$ are parametrized (canonically) by the conjugacy classes in the Weyl group $W$ of $G$. Let $\left\{T_{w} \mid w \in W / \sim\right\}$ be a set of representatives of these conjugacy classes. Thus the Green functions $Q_{T}^{G}(v)$ may be written $Q_{w}(v)=Q_{T_{w}}^{G}(v) \quad\left(v \in G_{\text {uni }}^{F}\right)$.

The group $Z_{G}(u)$ acts on $\mathscr{B}_{u}$ and induces an action of $C(u)$ on $\mathscr{B}_{u}$ and hence on the cohomology $H^{*}\left(\mathscr{B}_{u}\right)$.

The connection between the cohomology and representation theory arises from

(1.1) Theorem (Kazhdan, Springer, Shoji, Spaltenstein [10, 25, 18, 24]). Let notation be as above with $u \in G^{F}$ split, and $p$ and $q$ sufficiently large. 
(i) There is an action of $W$ on the (l-adic) cohomology $H^{*}\left(\mathscr{B}_{u}\right)$ (the Springer action as defined by Lusztig) such that for any $c \in C(u), w \in W$

$$
Q_{w}\left(u_{c}\right)=\sum_{i \geq 0} \operatorname{Tr}\left(w . c, H^{2 i}\left(\mathscr{B}_{u}\right)\right) q^{i} \quad\left(\text { and } H^{2 i+1}\left(\mathscr{B}_{u}\right)=0 \text { for all } i\right) .
$$

(ii) If $A$ is a nilpotent element of $\mathscr{G}_{\mathrm{c}}$ corresponding (see above) to $u \in G_{p}$, there is an action of $W$ on $H^{*}\left(\mathscr{B}_{A}\right)$ and $H^{*}\left(\mathscr{B}_{A}\right) \cong H^{*}\left(\mathscr{B}_{u}\right)$ as complex (or $\left.\overline{\mathbb{Q}}_{l}\right) W$-modules (see $\left.[20,85.3]\right)$. In particular, for $u$ split in $G^{F}$, we have

$$
Q_{w}(u)=\sum_{i \geq 0} \operatorname{Tr}\left(w, H^{2 i}\left(\mathscr{B}_{A}\right)\right) q^{i}
$$

In the statement of (1.1), we use Lusztig's construction [13] of the "Springer action" of $W$ on $H^{i}\left(\mathscr{B}_{A}, \mathbb{C}\right)$, which differs slightly from Springer's original definition (cf. Shoji $[19, \S 5]$ ). Moreover Lusztig's construction applies for an arbitrary element $A$ of $\mathscr{G}=\operatorname{Lie} G$. The formulae in Theorem (1.1) may be viewed as statements about a fixed $G$ for varying $q$. Thus it makes sense to say that for split $u, Q_{w}(u) \in \mathbb{Z}[q]$.

(1.2) Definition. With $G$ as above, let $A \in \mathscr{G}$. Write

$$
Q_{A}=\sum_{i \geq 0} H^{2 i}\left(\mathscr{B}_{A}, \mathbb{C}\right) q^{i} \in R(W)[q]
$$

where $R(W)$ is the Grothendieck ring of $W$ and $q$ is regarded as a variable in the sense of the above remark.

In this work we address the problem of determining the polynomials $Q_{A}$.

(1.3) Problem. Let $G$ be as above, $A \in \mathscr{G}$, and suppose $\chi \in \widehat{W}$. Compute the inner product

$$
\left\langle Q_{A}, \chi\right\rangle=\sum_{i \geq 0}\left\langle H_{i}^{2 i}\left(B_{A}, \mathbb{C}\right), \chi\right\rangle q^{i} \in \mathbb{Z}_{\geq 0}[q] .
$$

In particular we obtain an explicit formula for $\left\langle Q_{A}, \rho\right\rangle$ in the case where $A$ is a regular nilpotent element in a Levi subalgebra of a parabolic subalgebra of $\mathscr{G}$ (i.e. $A$ is of "parabolic type") and $\rho$ is the reflection character. Our formula is in terms of the geometry of hyperplane complements (see Section 2 below) and applies to all the classical groups, except for a possible ambiguity in the case of $S O(4 n+2)$.

The existence of such a formula was first observed empirically by Orlik, Solomon and Spaltenstein for the exceptional groups and subsequently formulated as a conjecture by Spaltenstein [23].

The proof of the main theorem is essentially case by case. No direct connection is established between the geometry of $\mathscr{B}_{A}$ and the Coxeter arrangement corresponding to $G$, although we make some speculations below. The 
basic tool is an extension to a theorem of Borho and MacPherson (see Section 3 below) which may be applied (Section 4) to give inequalities for $\left\langle Q_{A}, \rho\right\rangle$ which are just sufficient for its determination. All cases except for the orthogonal groups are relatively straightforward, while the orthogonal case requires the detailed computation of the cohomology with compact supports of some subvarieties of complex projective space, which is carried out in Section 5.

In Section 2 we discuss hyperplane complements and state the main theorem, and in Section 6 we complete its proof for the linear and symplectic cases, using the inequalities of Section 4 , which in the symplectic case yield $\left\langle Q_{A}, \rho\right\rangle$ for any nilpotent $A$, not only $A$ of parabolic type.

In Section 7 we treat the orthogonal groups (type $B$ and $C$ ) and complete the proof of the main theorem. Also in Section 7 we apply "Ennola duality of Green functions" (cf. [18], [24]) to determine $\left\langle Q_{A}, \rho\right\rangle$ for any nilpotent element $A \in \mathscr{G}$ when $G$ is of type $B_{n}(G=S O(2 n+1))$ or $D_{2 n} \quad(G=$ $S O(4 n))$. This method does not apply to $S O(4 n+2)$, but those of Section 4 do, and we are able to deal with $A$ of parabolic type in $S O(4 n+2)$ except for an ambiguity in the case (*) (see (2.4) below and (7.24) and (7.25).)

Finally in Section 8 we make some remarks concerning interpretation of the results of this work and conjecture a similar result for the compounds of the reflection representation.

\section{Hyperplane complements-Statement of the main theorem}

Maintaining the above notion, we let $l$ be the rank of $W$ (or semisimple rank of $G$ ) and let $\mathscr{A}$ be the Coxeter arrangement (in the sense of Orlik and Solomon [16]) consisting of the complexified reflecting hyperplanes of $W$ in $\mathbb{C}^{l}$. Let $L(W)$ be the lattice of intersections of hyperplanes in $\mathscr{A}$, ordered by the reverse of inclusion. For any element $X \in L(W)$ let $\mathscr{A}^{X}$ be the arrangement of hyperplanes of $X$ given by

$$
\mathscr{A}^{X}=\{X \cap H \mid H \in \mathscr{A}, X \not \subset H\} .
$$

Let $M_{X}$ be the corresponding hyperplane complement:

$$
M_{X}=X-\bigcup_{Y \in \mathscr{A}^{X}} Y \text {. }
$$

The empty hyperplane intersection is $\mathbb{C}^{l} \in L(W)$, and we write $M_{\mathbb{C}^{l}}=$ $M_{W}$, the usual hyperplane complement studied in [17] and [12].

(2.1) Theorem (Orlik and Solomon [16]). With the above notation, let $X \in L(W)$. Consider the Poincaré series $P_{X}(q)=\sum_{i \geq 0} \operatorname{dim}_{\mathbb{C}} H^{i}\left(M_{X}, \mathbb{C}\right) q^{i}$, 
where $H^{i}$ denotes ordinary (de Rham) cohomology. Then

$$
P_{X}(q)=\prod_{j=1}^{\operatorname{dim} X}\left(1+b_{j}(X) q\right) \quad \text { for certain integers } b_{j}(X) \in \mathbb{N} \text {. }
$$

Furthermore Orlik and Solomon give the integers $b_{j}(X)$ explicitly.

The polynomials $P_{X}(q)$ are related to the polynomials $P_{w}(t) \quad(w \in W)$ which were discussed in [12].

The proof of $(2.1)$ is case by case except when $X=\mathbb{C}^{l}$ or $X=H \in \mathscr{A}$. In the former case, we have

(2.2) $P_{\mathbf{C}^{\prime}}(q)=\left(1+m_{1} q\right)\left(1+m_{2} q\right) \cdots\left(1+m_{l} q\right)$, where $\left\{m_{1}, \ldots, m_{l}\right\}$ are the exponents of $W$, while if $X=H \in \mathscr{A}$, we have

(2.3) $P_{H}(q)=\left(1+m_{1} q\right)\left(1+m_{2} q\right) \cdots\left(1+m_{l-1} q\right)$, where $\left\{m_{1}, \ldots, m_{l}\right\}$ are the exponents of $W$, written in ascending order.

The results (2.2) and (2.3) are due to Arnol'd and Brieskorn [4] and Orlik, Solomon and Terao, respectively, 'On Coxeter arrangements and the Coxeter number', Adv. Stud. Pure Math. 8 (1987), 461-477.

Our main result is the following.

(2.4) Theorem. Suppose $G$ is a connected reductive complex Lie group with Borel subgroup $B$, Weyl group $W$ and corresponding set of simple roots $\Pi$. Let $J$ be a subset of $\Pi$ and suppose $A_{J}$ is a regular nilpotent element in the standard Levi subalgebra $\operatorname{Lie}\left(L_{J}\right)$ of the parabolic subalgebra $\operatorname{Lie}\left(P_{J}\right)$ of $\mathscr{G}=\operatorname{Lie} G$. Write $X_{J}=\bigcap_{\alpha \in J} \alpha^{\perp}$ (where $\alpha^{\perp}$ is the complex hyperplane corresponding to $\alpha$ in the Coxeter arrangement $\mathscr{A}$ of $W$ ). Then using the above notation, we have

$$
\left\langle Q_{A_{J}}, \rho\right\rangle=\sum_{j \geq 0} q^{b_{j}\left(X_{J}\right)}
$$

except possibly in the case (*) (see below).

Here, $Q_{A}=\sum_{i \geq 0} H^{2 i}\left(\mathscr{B}_{A}, \mathbb{C}\right) q^{i}$ for $A \in \mathscr{G}$ is the Green polynomial discussed in Section 1 . $\left(Q_{A} \in R(W)[q]\right)$ and $\rho$ is the reflection representation of $W$.

The excluded case is

$$
\begin{aligned}
& G=S O(4 n+2)\left(\text { type } D_{2 n+1}\right) \text { and } J \text { is a parabolic subsystem } \\
& \text { of type } A_{i_{1}} \times A_{i_{2}} \times \cdots \times A_{i_{k}} \text { with } 2 \sum_{j} i_{j}<2 n+1 .
\end{aligned}
$$

For further details concerning the ambiguity in the excluded case, see Section 7 below (in particular (7.24) and (7.25)). 
The case $J=\Pi$ is well known. It asserts (cf. (2.2) above) that if $\mathscr{B}=$ $G / B$

$$
\left\langle\sum_{i=0}^{\operatorname{dim} \mathscr{B}} H^{2 i}(\mathscr{B}, \mathbb{C}) q^{i}, \rho\right\rangle=\sum_{j=0}^{l} q^{m_{j}} .
$$

But $H^{*}(\mathscr{B}, \mathbb{C})$ is well known: it is the (graded) regular representation $(S / I)$ of $W$ where $S$ is the symmetric algebra on $V$ (the real representation space for $W$ ) and $I$ is the ideal generated by the $W$-invariants of positive degree (Borho-MacPherson [2], cf. Shoji [19]). Thus (2.5) reduces to a result of Solomon [21] in invariant theory.

For the exceptional groups, (2.4) was observed empirically by Solomon and Spaltenstein [20]; thus our theorem completes the proof of the corresponding statement for all groups, except for the ambiguity $(*)$.

The proof of $(2.4)$ proceeds essentially in a case by case fashion; that is, the two sides of the equation are computed and shown to be equal. However the computation of the left hand side is to a certain extent common to all classical groups $G$ (cf. Sections 3, 4 below). Since we shall require the right hand side of (2.4) explicitly we give it here.

(2.6) Proposition. Suppose $\mathscr{A}$ is of type $A_{l}, B_{l}$ or $C_{l}$ and that $J \subseteq \Pi$. Then $\mathscr{A}^{X_{J}}$ depends only on the cardinality $|J|$. Explicitly $\mathscr{A}^{X_{J}}$ is of the same type as $\mathscr{A}$, and has rank $|\Pi|-|J|$.

This may be found in Orlik and Solomon $[16, \S 2]$ and is perfectly straightforward to prove. It shows that the integers $b_{j}\left(X_{J}\right)$ are the exponents of Coxeter groups of lower rank, and so are known.

To discuss type $D_{l}$, we need the arrangements $D_{l}^{k}(k \leq l)$ defined as follows. Let $V$ be an Euclidean space and let $x_{1}, \ldots, x_{l}$ be a basis of $V^{*}$, dual to an orthonormal basis of $V$. Then $W$ (of type $D_{l}$ ) has corresponding arrangement $\left\{H_{i j}^{+}, H_{i j}^{-} \mid 1 \leq i<j \leq l\right\}$ where $H_{i j}^{+}=\operatorname{ker}\left(x_{i}-x_{j}\right), H_{i j}^{-}=$ $\operatorname{ker}\left(x_{i}+x_{j}\right)$. Let $H_{i}=\operatorname{ker} x_{i}$ and define (for $0 \leq k \leq l$ )

$$
D_{l}^{k}=\left\{H_{1}, \ldots, H_{k}, H_{i j}^{+}, H_{i j}^{-} \mid 1 \leq i<j \leq l\right\} .
$$

Thus $D_{l}^{0}=D_{l}$, while $D_{l}^{l}=B_{l}$.

(2.7) LemMA [17, (2.5)]. If $\mathscr{A}$ is the complex hyperplane arrangement $D_{l}^{k}$, then the Poincare polynomial of the corresponding hyperplane complement is given by

$$
P_{\mathscr{A}}(q)=(1+q)(1+3 q) \cdots(1+(2 l-3) q)(1+(k+l-1) q) .
$$


Now suppose $J \subseteq \Pi$ (type $D_{l}$ ). Then $J$ is either of type $A_{i_{1}} \times \cdots \times A_{i_{k}}$ with $\sum_{j=1}^{k}\left(i_{j}+1\right) \leq l$, or $A_{i_{1}} \times \cdots \times A_{i_{k}} \times D_{m}, m \geq 2, \sum_{j=1}^{k}\left(i_{j}+1\right)+$ $m \leq l$. Here we use the following notation: $\Pi=\left\{\alpha_{1}, \ldots, \alpha_{l}\right\}$, where $\alpha_{i}^{\perp}=H_{i, i+1}^{+} \quad(1 \leq i \leq l-1)$ and $\alpha_{n}^{\perp}=H_{n-1, n}^{-} ; D_{m}$ is a subsystem of type $\left\{\alpha_{n}, \alpha_{n-1}, \alpha_{n-2}, \ldots, \alpha_{n-m+1}\right\} \quad(m \geq 2)$.

(2.8) Proposition. Let $\mathscr{A}$ be an arrangement of type $D_{l}$ and suppose $J \subseteq \Pi$.

(i) If $J$ is of type $A_{i_{1}} \times \cdots \times A_{i_{k}}\left(i_{j} \geq 1\right)$, then $\mathscr{A}^{X}$ is of type $D_{|\Pi|-|J|}^{k}=$ $D_{l-|J|}^{k}$.

(ii) If $J$ is of type $A_{i_{1}} \times \cdots \times A_{i_{k}} \times D_{m}(m \geq 2)$, then $\mathscr{A}^{X_{J}}$ is of type $B_{|\Pi|-|J|}=B_{l-|J|}$

Proof. (i) We may take $J$ to be $\left\{\alpha_{1}, \ldots, \alpha_{i_{1}}, \alpha_{i_{1}+2}, \ldots, \alpha_{i_{1}+i_{2}+1}, \ldots\right.$, $\left.\alpha_{i_{1}+i_{2}+\cdots+i_{k}+k-1}\right\}$ in which case $X_{J}=\left\{\left(x_{1}, \ldots, x_{l}\right) \in V \mid x_{1}=\cdots=x_{i_{1}+1}\right.$, $\left.x_{i_{1}+2}=\cdots=x_{i_{1}+i_{2}+1}, \ldots\right\}$. Take coordinates $y_{1}\left(=x_{1}\right), y_{2} \quad\left(=x_{i_{1}+2}\right)$, $\ldots, y_{k}, x_{l}, x_{l-1}, \ldots, x_{d+1}$ in $X_{J}$, where $d=\sum_{j=1}^{k}\left(i_{j}+1\right)$. Then the restriction to $X_{J}$ of the linear form $x_{i}+x_{j}$ is $2 y_{m}$ for appropriate $i, j$ (any $m, 1 \leq m \leq k$ ). All other restrictions are of the form $H_{i j}^{+}$or $H_{i j}^{-}$, which proves (i).

(ii) Clearly $\alpha_{n}^{\perp} \cap \alpha_{n-1}^{\perp}=\left\{\left(x_{1}, \ldots, x_{l-2}, 0,0\right) \in V\right\}$; the restriction of $H_{i l}$ to $\alpha_{n}^{\perp} \cap \alpha_{n-1}^{\perp}$ is the same as that of $H_{i}$. It follows easily that in this case $\mathscr{A}^{X_{J}}=B_{\mathrm{dim} X_{J}}$.

We summarise the results of (2.6) and (2.8) explicitly in

(2.9) Proposition. Let $\mathscr{A}$ be a complete Coxeter arrangement of classical type. Let $J$ be a subset of some simple system of roots corresponding to $\mathscr{A}$. Then the integers $b_{i}\left(X_{J}\right)$ are given as follows:

(i) If $\mathscr{A}$ is of type $A_{l},\left\{b_{i}\left(X_{J}\right)\right\}=\{1,2, \ldots, l-|J|\}$.

(ii) If $\mathscr{A}$ is of type $B_{l}$ or $C_{l},\left\{b_{i}\left(X_{J}\right)\right\}=\{1,3,5, \ldots, 2 l-2|J|-1\}$.

(iii) If $\mathscr{A}$ is of type $D_{l}$ then

$$
\left\{b_{i}\left(X_{J}\right)\right\}=\left\{\begin{array}{c}
\{1,3,5, \ldots, 2 l-2|J|-1\} \\
\quad \text { if } J \text { has a component of type } D_{m}(m \geq 2) \\
\{1,3,5, \ldots, 2 l-2|J|-3, k+l-|J|-1\} \\
\text { if } J \text { has } k \text { components, each of type } A_{i}, \text { some } i .
\end{array}\right.
$$




\section{An equivariant exact sequence in cohomology}

The basic tool we shall use is the exact sequence for cohomology with compact supports [8]: if $X$ is a locally compact Hausdorff space and $Y$ is closed in $X$, then we have an exact sequence induced by the inclusion $Y \hookrightarrow X$ as follows:

$$
\cdots \rightarrow H_{c}^{i}(X) \rightarrow H_{c}^{i}(Y) \rightarrow H_{c}^{i+1}(X-Y) \rightarrow H_{c}^{i+1}(X) \rightarrow H_{c}^{i+1}(Y) \rightarrow \ldots
$$

where $H_{c}^{i}$ denotes complex valued cohomology with compact supports.

Now take $X=\mathscr{B}, Y=\mathscr{B}_{A}$. Then there is an action of $W$ on $H^{*}(\mathscr{B})$ and on $H^{*}\left(\mathscr{B}_{A}\right)$ (the "Springer action" as defined by Lusztig; see below).

(3.2) Proposition. (Hotta and Springer; Invent. Math. 41 (1977)). The maps $H^{i}(\mathscr{B}) \rightarrow H^{i}\left(\mathscr{B}_{A}\right)$ induced by the induction $\mathscr{B}_{A} \hookrightarrow \mathscr{B}$ are equivariant with respect to the Springer and Lusztig $W$-action.

For a proof, see Spaltenstein [22].

(3.3) Proposition. For any $A \in \mathscr{G}$, we have $H^{i}\left(\mathscr{B}_{A}\right)=0$ if $i$ is odd.

Combining (3.2) and (3.3), we obtain

(3.4) LEMMA. There is a canonically defined action of $W$ on $H_{c}^{*}\left(\mathscr{B}-\mathscr{B}_{A}\right)$ which makes the sequence (3.1) $W$-equivariant (where $X=\mathscr{B}, Y=\mathscr{B}_{A}$ ) if the $W$-action on $H^{*}(\mathscr{B})$ and $H^{*}\left(\mathscr{B}_{A}\right)$ is the Springer and Lusztig action.

Proof. By (3.3), we have for each $i$ an exact sequence

$$
0 \rightarrow H_{c}^{2 i}\left(\mathscr{B}-\mathscr{B}_{A}\right) \stackrel{\alpha}{\rightarrow} H^{2 i}(\mathscr{B}) \stackrel{\beta}{\rightarrow} H^{2 i}\left(\mathscr{B}_{A}\right) \stackrel{\gamma}{\rightarrow} H_{c}^{2 i+1}\left(\mathscr{B}-\mathscr{B}_{A}\right) \rightarrow 0
$$

where by (3.2), the map $\beta$ is $W$-equivariant. Hence $\operatorname{ker} \beta=\operatorname{im} \alpha$ is $W$ stable, and since $\alpha$ is a monomorphism, there is a unique action of $W$ on $H_{c}^{2 i}\left(\mathscr{B}-\mathscr{B}_{A}\right)$ defined by the $W$-action on $\operatorname{im} \alpha$. Similarly the requirement of $W$-equivariance defines a unique $W$-action on $H_{c}^{2 i+1}\left(\mathscr{B}-\mathscr{B}_{A}\right)=\operatorname{im} \gamma$.

Our aim is to compute the $\rho$-isotypic part of the sequence (3.4.1). This will be achieved by taking the $W_{1}$-invariant part of (3.4.1) for $W_{1}$ an appropriate subgroup of $W$ and using the simple

(3.5) LemMA. Let $M$ be a $\mathbb{C W}$-module and let $W_{1}$ be a subgroup of $W$. Write $\operatorname{Ind}_{W_{1}}^{W}(1)=\sum_{i=0}^{k} m_{i} \xi_{i} \quad\left(\xi_{i}\right.$ irreducible). Then $\operatorname{dim}_{\mathrm{C}} M^{W_{1}}=$ $\sum_{i=1}^{k} m_{i}\left\langle M, \xi_{i}\right\rangle$. (Here $M^{W_{1}}$ denotes $\left\{m \in M \mid w m=m \forall w \in W_{1}\right\}$.) 
The subgroup $W_{1}$ will be the Weyl group $W(L)$ of a Levi subgroup $L$ of an appropriate parabolic subgroup $P \supseteq B$ of $G$. Let $P=L U$ be a Levi decomposition of $P$, with $L \supseteq T$. The computation of the $W(L)$-invariant part of (3.4.1) has been partly carried out by Borho and MacPherson [3, $\S \S(2.8),(3.4)]$.

(3.6) Proposition (Borho and MacPherson [2]). With the above notation write $\mathscr{P}=G / P, \mathscr{P}_{A}=\{x P \in \mathscr{P} \mid A \in \operatorname{Adx}(\operatorname{Lie} P)\}$. Then we have isomorphisms

$$
H^{i}\left(\mathscr{B}_{A}\right)^{W(L)} \simeq H^{i}\left(\mathscr{P}_{A}\right)
$$

This gives the $W(L)$-invariant part of two of the four non-trivial terms in (3.4.1). To obtain the others we require the following version of the Borho and MacPherson result (for which we are grateful to the referee).

(3.7) Proposition. With notation as in (3.6), the natural map $\mathscr{B}_{A} \rightarrow \mathscr{P}_{A}$ $(x B \mapsto x P)$ induces for each $i$, a homomorphism $H^{i}\left(\mathscr{P}_{A}\right) \rightarrow H^{i}\left(\mathscr{B}_{A}\right)$. This is a monomorphism with image $H^{i}\left(\mathscr{B}_{A}\right)^{W(L)}$.

To see (3.7), we shall need to recall the Lusztig construction of the $W$ action on $H^{*}\left(\mathscr{B}_{A}\right)$. Before doing this, however, we set out some consequences of (3.7).

(3.8) Proposition. With notation as above, we have diagrams

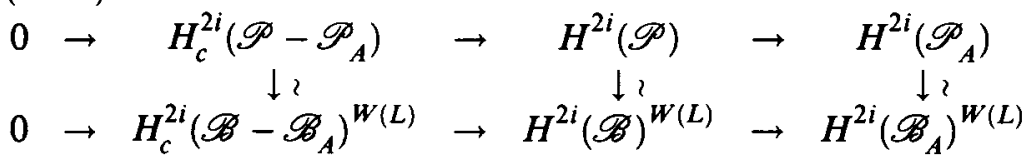

$$
\begin{aligned}
& \rightarrow \quad H_{c}^{2 i+1}\left(\mathscr{P}-\mathscr{P}_{A}\right) \rightarrow 0 \\
& \rightarrow H_{c}^{2 i+1}\left(\mathscr{B}-\mathscr{B}_{A}\right)^{W(L)} \rightarrow 0
\end{aligned}
$$

where the vertical arrows are isomorphisms and are induced by the projection $\mathscr{B} \rightarrow \mathscr{P}$ and its various restrictions. Moreover the squares are all commutative.

Proof. Since $H^{i}\left(\mathscr{P}_{A}\right) \cong H^{i}\left(\mathscr{B}_{A}\right)^{W(L)}$, it follows from (3.2) that $H^{i}\left(\mathscr{P}_{A}\right)$ vanishes for $i$ odd and hence the top row of the diagram (3.8.1) is just a part of the cohomology long exact sequence of the pair $\left(\mathscr{P}, \mathscr{P}_{A}\right)$. Now it follows by the functoriality of the cohomology long exact sequence of a pair, applied 
to the map of pairs defined by $\left(\mathscr{B}, \mathscr{B}_{A}\right) \rightarrow\left(\mathscr{P}, \mathscr{P}_{A}\right)$ (the projection), that we have a diagram

$$
\begin{aligned}
& 0 \rightarrow H_{c}^{2 i}\left(\mathscr{P}-\mathscr{P}_{A}\right) \rightarrow H^{2 i}(\mathscr{P}) \rightarrow H^{2 i}\left(\mathscr{P}_{A}\right) \rightarrow H^{2 i+1}\left(\mathscr{P}-\mathscr{P}_{A}\right) \rightarrow 0 \\
& \downarrow \beta_{1} \quad \downarrow \beta_{2} \quad \downarrow \beta_{3} \quad \downarrow \beta_{4} \\
& 0 \rightarrow H_{c}^{2 i}\left(\mathscr{B}-\mathscr{B}_{A}\right) \rightarrow H^{2 i}(\mathscr{B}) \rightarrow H^{2 i}\left(\mathscr{B}_{A}\right) \rightarrow H_{c}^{2 i+1}\left(\mathscr{B}-\mathscr{B}_{A}\right) \rightarrow 0
\end{aligned}
$$

in which the vertical arrows are induced by the various restrictions of the projection $\mathscr{B} \rightarrow \mathscr{P}$, the rows are part of the respective cohomology exact sequences and each small square is commutative.

By (3.7), $\beta_{2}$ and $\beta_{3}$ are isomorphisms onto the $W(L)$-invariant parts of $H^{2 i}(\mathscr{B})$ and $H^{2 i}\left(\mathscr{B}_{A}\right)$ respectively. It is now straightforward to deduce that $\beta_{1}$ and $\beta_{4}$ are isomorphisms onto the $W(L)$-invariant parts of $H_{c}^{2 i}\left(\mathscr{B}-\mathscr{B}_{A}\right)$ and $H_{c}^{2 i+1}\left(\mathscr{B}-\mathscr{B}_{A}\right)$ respectively. Hence we may take the $W$-invariant part of the second row of (3.8.2) to obtain the diagram (3.8.1).

We have as an immediate consequence

(3.9) Corollary. With the $W$-action on $H_{c}^{*}\left(\mathscr{B}-\mathscr{B}_{A}\right)$ as defined in (3.4), we have, for each $i$, an isomorphism

$$
H_{c}^{i}\left(\mathscr{P}-\mathscr{P}_{A}\right) \stackrel{\sim}{\rightarrow} H_{c}^{i}\left(\mathscr{B}-\mathscr{B}_{A}\right)^{W(L)}
$$

induced by the projection $\mathscr{B}-\mathscr{B}_{A} \rightarrow \mathscr{P}-\mathscr{P}_{A}$.

We now turn to a discussion of (3.7) and (3.2). For this we recall the construction of Lusztig ([13], see also [19, $\S \S 4,5])$ and the proof of Borho and MacPherson.

With the above notation, let $\tilde{\mathscr{G}}=\{(x, g B) \in \mathscr{G} \times \mathscr{B} \mid x \in \operatorname{Adg}$ Lie $B\}$. Then the projection to the first coordinate $\pi: \tilde{\mathscr{G}} \rightarrow \mathscr{G}$ is the well-known "Grothendieck resolution", while the projection to the second coordinate is a fibration with fibre $\operatorname{Lie} B$, which shows that $H^{*}(\tilde{\mathscr{G}}) \simeq H^{*}(\mathscr{B})$. Now let $\mathscr{G}_{\mathrm{rs}}$ be the set of regular semisimsple elements of $\mathscr{G}$ and write $\widetilde{\mathscr{G}}_{\mathrm{rs}}=\pi^{-1}\left(\mathscr{G}_{\mathrm{rs}}\right)$. Then $\pi_{\mathrm{rs}}=\left.\pi\right|_{\tilde{\mathscr{F}}_{\mathrm{rs}}}: \tilde{\mathscr{G}}_{\mathrm{rs}} \rightarrow \mathscr{G}_{\mathrm{rs}}$ is an unramified covering with Galois group $W$; in fact $\pi_{\mathrm{rs}}^{-1}(x)=\{g w B \mid w \in W\}$, where $g B$ is a fixed element of $\mathscr{B}$ such that $x \in \operatorname{Adg} \operatorname{Lie} B \quad\left(x \in \mathscr{G}_{\mathrm{rs}}\right)$. It follows that if $\mathbb{C}$ is the constant sheaf on $\widetilde{\mathscr{G}}_{\mathrm{rs}}$, then $\mathscr{L}=\left(\pi_{\mathrm{rs}}\right)_{*} \mathbb{C}$ is a locally constant sheaf (that is, a local system) on $\mathscr{G}_{\mathrm{rs}}$ which admits $W$ as a group of sheaf automorphisms. The key to Lusztig's construction is

(3.10) THEOREM (Lusztig [13]). With the above notation, we have

$$
\mathrm{IC}(\mathscr{G}, \mathscr{L}) \simeq \mathbb{R} \pi_{*} \mathbb{C}
$$


where IC denotes the intersection complex functor in $\mathscr{D}_{c}^{b}(\mathscr{G})$ and $\mathbb{C}$ (on the right) denotes the constant sheaf on $\tilde{\mathscr{G}}$.

The proof of (3.10) is achieved by characterising a shift of the complex on the right as the perverse sheaf the cohomology of whose restriction to $\mathscr{G}_{\text {rs }}$ coincides with $\mathscr{L}$.

The Lusztig definition of the Springer action is now clear. By the functorial nature of IC, the left side of (3.10) admits $W$ as a group of automorphisms (in $\mathscr{D}_{c}^{b}(\mathscr{G})$ ); hence the same is true of the right side.

Now apply the cohomology functor and take the stalk at $A \in \mathscr{G}$ to deduce that there is a $W$-action on $\mathscr{H}^{i}\left(\mathbb{R} \pi_{*} \mathbb{C}\right)_{A} \cong H^{i}\left(\pi^{-1}(A), \mathbb{C}\right)=H^{i}\left(\mathscr{B}_{A}\right)$ since $\pi$ is proper. This is the "Springer action" we are concerned with.

Now let $P=L U$ be a parabolic subgroup as in (3.4). Write $\widehat{\mathscr{G}}=$ $\{(x, g P) \in \mathscr{G} \times \mathscr{P} \mid x \in$ Adg Lie $P\}$ and define $\gamma: \tilde{\mathscr{G}} \rightarrow \hat{\mathscr{G}}$ by $\gamma(x, g B)=$ $(x, g P)$ and $\xi: \hat{\mathscr{G}} \rightarrow \mathscr{G}$ by $\xi(x, g P)=x$. Then we have a commutative diagram

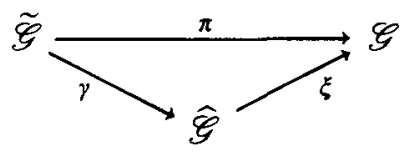

which induces

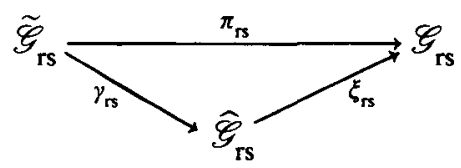

where $\xi_{\mathrm{rs}}, \hat{\mathscr{G}}_{\mathrm{rs}}$ and $\gamma_{\mathrm{rs}}$ are defined in the obvious way. Borho and MacPherson observe $[2, \S 2.5]$ that $\gamma_{\mathrm{rs}}$ is also a covering projection, this time with group $W(L)$. They deduce from this $[2, \S 2.7]$ that

(3.11) Proposition (Borho and MacPherson). With the above notation, we have $\mathbb{R} \xi_{*} \mathbb{C}=\left(\mathbb{R} \pi_{*} \mathbb{C}\right)^{W(L)}$ in $\mathscr{D}_{c}^{b}(\mathscr{G})$.

Applying the cohomology functor and taking stalks at $A \in \mathscr{G}$ gives (3.6).

(3.12) Proof of (3.7). We recall the following standard results in homological algebra. If $f: X \rightarrow Y$ is a continuous map of locally compact Hausdorff topological spaces, then there is a natural isomorphism of functors (cf. Hartshorne [9, Proposition 5.2])

$$
\mathbb{R} \Gamma_{Y} \circ \mathbb{R}_{*} \stackrel{\sim}{\rightarrow} \Gamma_{X}
$$

where $\Gamma_{X}$ and $\Gamma_{Y}$ denote the global section functors (this is just the Leray 
spectral sequence). Taking cohomology of both sides of (3.12.1), we obtain (3.12.2) For $\mathscr{F} \in \mathscr{D}_{c}^{b}(X)$, we have a natural isomorphism

$$
\mathbb{H}^{i}\left(Y, \mathbb{R} f_{*} \mathscr{F}\right) \rightarrow \mathbb{H}^{i}(X, \mathscr{F}) \text {. }
$$

Next, maintaining the above notation, it follows from the fact that $\mathbb{R} f_{*}$ is an adjoint of $f^{*}$, that for $\mathscr{F} \in \mathscr{D}_{c}^{b}(Y)$ we have

$$
\operatorname{Mor}_{\mathscr{D}_{c}^{b}(X)}\left(f^{*} \mathscr{F}, f^{*} \mathscr{F}\right) \simeq \operatorname{Mor}_{\mathscr{D}_{c}^{b}(Y)}\left(\mathscr{F}, \mathbb{R} f_{*} f^{*} \mathscr{F}\right) \text {. }
$$

Hence there is a distinguished element $\beta_{f} \in \operatorname{Mor}_{\mathscr{D}_{c}^{b}(X)}\left(\mathscr{F}, \mathbb{R} f_{*} f^{*} \mathscr{F}\right)$, namely the morphism corresponding to $\mathrm{id}_{f^{*} \mathscr{F}}$ on the left side of (3.12.3). This is called the "natural map": $\mathscr{F} \rightarrow \mathbb{R} f_{*} f^{*} \mathscr{F}$. Applying the cohomology functor, we get maps

$$
\mathbb{H}^{i}(Y, \mathscr{F}) \rightarrow \mathbb{H}^{i}\left(Y, \mathbb{R} f_{*} f^{*} \mathscr{F}\right) \underset{(3.12 .2)}{\stackrel{\sim}{\overrightarrow{1}}} \mathbb{H}^{i}\left(Y, f^{*} \mathscr{F}\right)
$$

The composite

$$
\mathbb{H}^{i}(Y, \mathscr{F}) \rightarrow \mathbb{H}^{i}\left(X, f^{*} \mathscr{F}\right)
$$

is called the map in cohomology which is naturally induced by $f$.

Now consider a commutative triangle

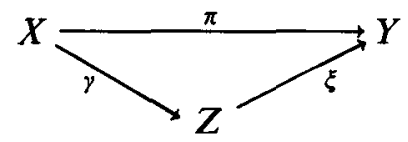

where $\pi, \xi, \gamma$ are proper maps of locally compact Hausdorff spaces.

For $\mathscr{F} \in \mathscr{D}_{c}^{b}(Z)$, apply the functor $\mathbb{R} \xi_{*}$ to the natural map $\mathscr{F} \rightarrow$ $\mathbb{R} \gamma_{*} \gamma^{*} \mathscr{F}$, obtaining

$$
\mathbb{R} \xi_{*} \mathscr{F} \rightarrow \mathbb{R} \xi_{*} \mathbb{R} \gamma_{*} \gamma^{*} \mathscr{F} \stackrel{\sim}{\rightarrow} \pi_{*} \gamma^{*} \mathscr{F}
$$

and taking stalks at $y \in Y$, we have maps

$$
\left(\mathbb{R} \xi_{*} \mathscr{F}\right)_{y} \simeq \mathbb{H}^{*}\left(\xi^{-1}(y), \mathscr{F}\right) \rightarrow\left(\mathbb{R} \pi_{*} \gamma^{*} \mathscr{F}\right)_{y} \simeq \mathbb{H}^{*}\left(\pi^{-1}(y), \gamma^{*} \mathscr{F}\right) \text {. }
$$

This map is precisely the map (3.12.4) in cohomology induced by the restriction $\gamma: \pi^{-1}(y) \rightarrow \xi^{-1}(y) \quad(\pi(x)=y$ implies $\gamma(x)=y)$.

Now consider the situation of (3.11). We have

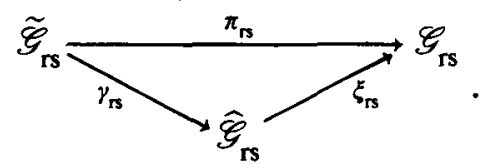

Take $\mathscr{F}$ to be the constant sheaf $\mathbb{C}$ in dimension 0 and zero elsewhere in (3.12.6). Then $\gamma_{\mathrm{rs}}^{*} \mathbb{C}=\mathbb{C}$ and from (3.12.6) we obtain the natural map

$$
\eta:\left(\xi_{\mathrm{rs}}\right)_{*} \mathbb{C} \rightarrow\left(\pi_{\mathrm{rs}}\right)_{*} \mathbb{C}
$$


and then clearly the image of $\eta$ is the $W(L)$-invariant part of $\left(\pi_{\mathrm{rs}}\right)_{*} \mathbb{C}$. Thus we have the commutative diagram

$$
\left(\xi_{\mathrm{rs}}\right)_{*} \mathbb{C} \overbrace{\left(\left(\pi_{\mathrm{rs}}\right) * \mathbb{C}\right)^{W(L)}} \underset{j}{\longrightarrow}\left(\pi_{\mathrm{rs}}\right)_{*} \mathbb{C}
$$

where $i$ is an isomorphism and $j$ is a monomorphism (cf. local systems on $\left.\mathscr{G}_{\mathrm{rs}}\right)$. Applying the functor IC to this diagram, we obtain

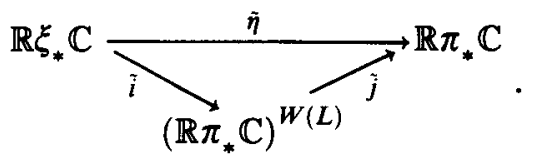

Moreover $\tilde{\eta}$ is precisely the map of (3.12.6), applied with $\mathscr{F}=\mathbb{C}$ to the diagram

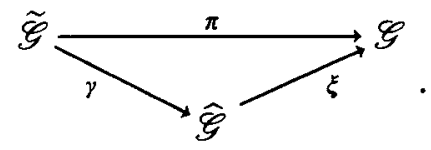

This is because $\mathbb{R} \xi_{*} \mathbb{C}$ and $\mathbb{R} \pi_{*} \mathbb{C}$ are semisimple perverse sheaves which are induced from local systems on $\mathscr{G}_{\text {rs }}$ by IC, from which it follows that any morphism: $\mathbb{R} \xi_{*} \mathbb{C} \rightarrow \mathbb{R} \pi_{*} \mathbb{C}$ is determined by its restriction to $\left.\mathbb{R} \xi_{*} \mathbb{C}\right|_{\mathscr{\xi}_{\mathrm{rs}}}$. Since our two maps clearly have the same restriction, they coincide.

Now taking stalks in (3.12.8) at $A \in \mathscr{G}$, we get

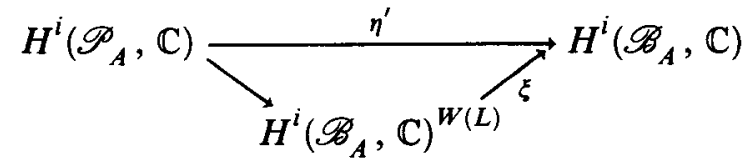

and $\eta^{\prime}$ is the map induced by $\gamma: \mathscr{B}_{A} \rightarrow \mathscr{P}_{A}$. This proves (3.7).

\section{Some basic inequalities}

In this section we develop briefly a "calculus" of polynomials whose coefficients are (virtual) representations of a finite group $H$. The method of proof of our main result depends on using exact sequences in cohomology to establish inequalities for $\left\langle H^{p}\left(\mathscr{B}_{A}\right), \rho\right\rangle$ which in general are sufficient for its determination. Our purpose here is to encode the inequalities in such a way as to make the argument mechanical in each case.

(4.1) Definition. Let $H$ be a finite group. Then $R(H)$ will denote the Grothendieck ring of isomorphism classes of complex (virtual) representations of $H$. We write $\hat{H}$ for the set of isomorphism classes of complex irreducible representations of $H$. 
Since $\mathbb{C}$-representations are completely reducible, the additive group of $R(H)$ is free abelian, with basis $\widehat{H}$. We have on $R(H)$ the usual inner product $\langle M, N\rangle=\langle M, N\rangle_{H}(M, N \in R(H))$ which makes $\hat{H}$ an orthonormal basis. If $q$ is an indeterminate, denote by $R(H)[q]$ the ring of polynomials in $q$, with coefficients in $R(H)$. Then we can extend to $R(H)[q]$ the operations on $R(H)$, as follows.

For a subgroup $K \leq H$ and $H$-module $A$, denote by $A^{K}$ the set of $K$ fixed points of $A$, and extend to $R(H)$ by linearity. Similarity if $\xi \in \widehat{H}$, $A_{\xi}$ denotes the $\xi$-isotypic part of $A$ and we extend to $R(H)$ in the obvious way.

(4.2) Definition. Let $Q=\sum_{i=0}^{n} M_{i} q^{i} \in R(H)[q]$, let $K$ be a subgroup of $H$ and let $N \in R(H)$ and $\xi \in \widehat{H}$. Then we define

$$
\langle Q, N\rangle=\sum_{i=0}^{n}\left\langle M_{i}, N\right\rangle q^{i} \in \mathbb{Z}[q] .
$$

$$
Q^{K}=\sum_{i=0}^{n} M_{i}^{K} q^{i} \in R\left(N_{H}(K)\right)[q]
$$

$$
Q_{\xi}=\sum_{i=0}^{n}\left(M_{i}\right)_{\xi} q^{i}
$$

$$
\operatorname{dim} Q=\sum_{i=0}^{n}\left(\operatorname{dim} M_{i}\right) q^{i}, \text { where } \operatorname{dim}: R(H) \rightarrow \mathbb{Z}
$$

is the dimension homomorphism.

Given polynomials $f=\sum a_{i} q^{i}, g=\sum b_{i} q^{i}$ in $\mathbb{Z}[q]$, we say $f \leq g$ if $a_{i} \leq b_{i}$ for each $i$. If $Q \in R(H)[q]$, we say $Q \geq 0$ if $\left\langle Q, \xi_{i}\right\rangle \geq 0$ for all $\xi_{i} \in \widehat{H}$.

(4.3) Lemma. With notation as in (4.2) above, suppose $Q \geq 0$; then we have

$$
\operatorname{dim} Q^{K}=\left\langle Q, 1_{K}^{H}\right\rangle, \text { where } 1_{K}^{H}=\operatorname{Ind}_{K}^{H}(1) .
$$

$$
\text { If } \xi \in \widehat{H} \text { and }\left\langle\xi, 1_{K}^{H}\right\rangle \neq 0 \text {, then }\langle Q, \xi\rangle \leq \operatorname{dim} Q^{K} \text {. }
$$

Proof. (i) For any $H$-module $M$, we have

$$
\operatorname{dim} M^{K}=\langle M, 1\rangle_{K}=\left\langle M, 1_{K}^{H}\right\rangle,
$$

the latter being Frobenius reciprocity. The result follows. 
(ii) From (i), $\operatorname{dim} Q^{K}=\left\langle Q, 1_{K}^{H}\right\rangle$; writing $1_{K}^{H}=\sum_{\xi_{i} \in H} m_{i} \xi_{i}$, we see $\operatorname{dim} Q^{K}=\sum_{\xi_{i} \in H} m_{i}\left\langle Q, \xi_{i}\right\rangle$. The result is now clear since $\left\langle Q, \xi_{i}\right\rangle \geq 0$ for all $i$.

Now recall the exact sequence (3.4.1) of $W$-modules:

$$
0 \rightarrow H_{c}^{2 i}\left(\mathscr{B}-\mathscr{B}_{A}\right) \rightarrow H^{2 i}(\mathscr{B}) \rightarrow H^{2 i}\left(\mathscr{B}_{A}\right) \rightarrow H_{c}^{2 i+1}\left(\mathscr{B}-\mathscr{B}_{A}\right) \rightarrow 0 .
$$

To express the exact nature of this sequence in terms of an equation in $R(W)[q]$, we make the following

(4.5) Definition (cf. Definition 5.1 below). Let $M$ be a complex variety whose cohomology $H_{c}^{*}(M)$ (with compact supports) has a $W$-action. Define

$$
\left.\begin{array}{l}
Q_{e}(M)=\sum_{i=0}^{\infty} H_{c}^{2 i}(M) q^{i} \\
Q_{o}(M)=\sum_{i=0}^{\infty} H_{c}^{2 i+1}(M) q^{i}
\end{array}\right\} \in R(W)[q] .
$$

Then $Q_{e}\left(\mathscr{B}_{A}\right)=Q_{A}$ (the Green polynomial of $A$ ), and from (4.4) it follows that

$$
Q_{e}\left(\mathscr{B}-\mathscr{B}_{A}\right)+Q_{e}\left(\mathscr{B}_{A}\right)=Q_{e}(\mathscr{B})+Q_{o}\left(\mathscr{B}-\mathscr{B}_{A}\right)
$$

(4.7) Proposition. With notation as in Section 3 and above, let $\chi \in \widehat{W}$ be an irreducible constituent of $\operatorname{Ind}_{W(L)}^{W}(1)$. Then $\left\langle Q_{0}, \chi\right\rangle-\operatorname{dim} Q_{e}\left(\mathscr{B}-\mathscr{B}_{A}\right)^{W(L)} \leq\left\langle Q_{A}, \chi\right\rangle \leq\left\langle Q_{0}, \chi\right\rangle+\operatorname{dim} Q_{o}\left(\mathscr{B}-\mathscr{B}_{A}\right)^{W(L)}$ where $Q_{0}=Q_{e}(\mathscr{B})=Q_{e}\left(\mathscr{B}_{0}\right)$.

Proof. Take inner products of both sides of (4.6) with $\chi$ :

$$
\left\langle Q_{e}\left(\mathscr{B}-\mathscr{B}_{A}\right), \chi\right\rangle+\left\langle Q_{A}, \chi\right\rangle=\left\langle Q_{0}, \chi\right\rangle+\left\langle Q_{o}\left(\mathscr{B}-\mathscr{B}_{A}\right), \chi\right\rangle,
$$

whence

$$
\left\langle Q_{A}, \chi\right\rangle=\left\langle Q_{0}, \chi\right\rangle+\left\langle Q_{o}\left(\mathscr{B}-\mathscr{B}_{A}\right), \chi\right\rangle-\left\langle Q_{e}\left(\mathscr{B}-\mathscr{B}_{A}\right), \chi\right\rangle .
$$

Moreover from (4.3)(ii), we have $\left\langle Q_{o}\left(\mathscr{B}-\mathscr{B}_{A}\right), \chi\right\rangle \leq \operatorname{dim} Q_{o}\left(\mathscr{B}-\mathscr{B}_{A}\right)^{W(L)}$ and $\left\langle Q_{e}\left(\mathscr{B}-\mathscr{B}_{A}\right), \chi\right\rangle \leq \operatorname{dim} Q_{e}\left(\mathscr{B}-\mathscr{B}_{A}\right)^{W(L)}$. The stated inequalities follow.

With $M$ as in (4.5), write (consistently with (5.1) below)

$$
P_{e}(M)=\operatorname{dim} Q_{e}(M), \quad P_{o}(M)=\operatorname{dim} Q_{o}(M) .
$$

For polynomials $f=\sum a_{i} q^{i}, g=\sum b_{i} q^{i} \in \mathbb{Z}[q]$, write

$$
\sup (f, g)=\sum \max \left(a_{i}, b_{i}\right) q^{i} \quad \text { and } \quad \inf (f, g)=\sum \min \left(a_{i}, b_{i}\right) q^{i} .
$$


Then we have

(4.8) Corollary. Let $A, P, L$ and $\chi \in \widehat{W}$ be as above (in 4.7)). Then (writing $\mathscr{P}=G / P$ etc. as in Section 3) we have

$$
\begin{aligned}
\sup & \left\{0,\left\langle Q_{0}, \chi\right\rangle-P_{e}\left(\mathscr{P}-\mathscr{P}_{A}\right)\right\} \leq\left\langle Q_{A}, \chi\right\rangle \\
& \leq \inf \left\{P_{e}\left(\mathscr{P}_{A}\right),\left\langle Q_{0}, \chi\right\rangle+P_{o}\left(\mathscr{P}-\mathscr{P}_{A}\right)\right\} .
\end{aligned}
$$

Proof. In addition to the inequality of (4.7), we have also $0 \leq\left\langle Q_{A}, \chi\right\rangle \leq$ $\operatorname{dim} Q_{A}^{W(L)}$. But by the Borho and MacPherson theorem (3.5), $\operatorname{dim} Q_{A}^{W(L)}$ $=P_{e}\left(\mathscr{P}_{A}\right)$ and further, by Corollary (3.7), $\operatorname{dim} Q_{e}\left(\mathscr{B}-\mathscr{B}_{A}\right)^{W(L)}=$ $P_{e}\left(\mathscr{P}-\mathscr{P}_{A}\right)$ and $\operatorname{dim} Q_{o}\left(\mathscr{B}-\mathscr{B}_{A}\right)^{W(L)}=P_{o}\left(\mathscr{P}-\mathscr{P}_{A}\right)$. Putting together the two inequalities with the above facts gives (4.8).

In practice, (4.8) is sufficient to determine $\left\langle Q_{A}, \rho\right\rangle$ for the linear and symplectic groups, while for the orthogonal groups we also require

(4.9) Proposition (cf. Alvis and Lusztig [1]). Suppose $A \in \mathscr{G}$ is parabolic of type $J \subset \Pi$ (that is, $A$ is a regular nilpotent element in a Levi subalgebra of a parabolic subalgebra of type $J)$. Then for $\chi \in \widehat{W},\left\langle Q_{A}, \chi\right\rangle_{q=1}=$ $\left\langle\operatorname{Ind}_{W_{J}}^{W}(1), \chi\right\rangle$ (that is, $\left.\left(Q_{A}\right)_{q=1}=\operatorname{Ind}_{W_{J}}^{W}(1)\right)$.

\section{Some cohomology computations}

Our purpose in this section is to assemble several results concerning the cohomology (with compact supports) of certain complex varieties which arise elsewhere in our discussion. The basic tool for the proofs of the results below is the exact sequence for cohomology with compact supports and since many of the proofs are similar, we shall suppress some details. In this section $\mathbf{A}^{k}$ (respectively $\mathbb{P}^{k}$ ) denotes $k$-dimensional complex affine (respectively projective) space. All cohomology is complex, with compact supports. Throughout this chapter we use without comment some obvious embeddings, such as $\mathbb{A}^{k-1} \hookrightarrow \mathbb{A}^{k}, \mathbb{P}^{k-1} \hookrightarrow \mathbb{P}^{k}$ etc.

In the interests of efficiency for the statements, and with a view to our applications, we make the following definitions.

(5.1) Definition. Let $M$ be a complex variety. Define

$$
P_{e}(M)=\sum_{i=0}^{\infty} \operatorname{dim} H_{c}^{2 i}(M) q^{i} \in \mathbb{Z}[q]
$$

and

$$
P_{o}(M)=\sum_{i=0}^{\infty} \operatorname{dim} H_{c}^{2 i+1}(M) q^{i} \in \mathbb{Z}[q]
$$


Thus the Poincaré series

$$
P(M)=\sum_{i=0}^{\infty} \operatorname{dim} H_{c}^{i}(M) q^{i / 2}=P_{e}(M)+q^{1 / 2} P_{o}(M) .
$$

The following lemma is basic and elementary.

(5.2) Lemma. (i) Suppose $X$ is a complex variety of (complex) dimension $k$ and $Y$ is a closed subvariety of dimension $r<k$, such that $H_{c}^{i}(X-Y)=0$ for $i<2 k$. Then

$$
H_{c}^{i}(X)=\left\{\begin{array}{l}
H_{c}^{i}(X-Y) \quad \text { if } i=2 k \\
H_{c}^{i}(Y) \quad \text { otherwise }(i<2 k)
\end{array}\right.
$$

$$
\begin{gathered}
P\left(\mathbb{A}^{k}\right)=P_{e}\left(\mathbb{A}^{k}\right)=q^{k} . \\
P\left(\mathbb{P}^{k}\right)=P_{e}\left(\mathbb{P}^{k}\right)=1+q+q^{2}+\cdots+q^{k} . \\
P\left(\mathbb{P}^{k}-\mathbb{P}^{l}\right)=P_{e}\left(\mathbb{P}^{k}-\mathbb{P}^{l}\right)=q^{l+1}+q^{l+2}+\cdots+q^{k} \quad(l<k) . \\
P_{e}\left(\mathbb{A}^{k}-\mathbb{A}^{l}\right)=q^{k}, \quad P_{o}\left(\mathbb{A}^{k}-\mathbb{A}^{l}\right)=q^{l} \quad(l<k) .
\end{gathered}
$$

(5.3) Proposition (Fary [7, Theorem 3, page 35]). Let $\mathscr{X}_{k}$ be the complex variety defined by $\mathscr{X}_{k}=\left\{\left(z_{1}, \ldots, z_{k}\right) \in \mathbb{A}^{k} \mid \sum_{i=1}^{k} z_{i}^{2}=1\right\}$. Then we have, for $k \geq 1, P\left(\mathscr{X}_{k}\right)=q^{(k-1) / 2}+q^{k-1}$.

(5.4) Corollary. Let

$$
\mathscr{P}_{k}=\left\{\left[z_{1}, \ldots, z_{k+1}\right] \in \mathbb{P}^{k} \mid \sum_{i=1}^{k+1} z_{i}^{2}=0\right\} \quad(k \geq 0) .
$$

Then the cohomology of $\mathscr{P}_{k}$ is given by

$$
\begin{gathered}
P_{o}\left(\mathscr{P}_{k}\right)=0, \quad P_{e}\left(\mathscr{P}_{2 k}\right)=1+q+\cdots+q^{2 k-1}, \\
P_{e}\left(\mathscr{P}_{2 k+1}\right)=1+q+\cdots+q^{2 k}+q^{k} .
\end{gathered}
$$

The corollary is proved by induction on $k$, using the exact sequence of the filtration $\mathscr{P}_{k} \supset \mathscr{P}_{k-1}, \mathscr{P}_{k}-\mathscr{P}_{k-1} \simeq \mathscr{X}_{k}$, together with (5.3).

We shall also require the following result of Fary. 
(5.5) Proposition (Fary [7, page 52]). Let $\mathscr{Y}_{k}=\left\{\left(z_{1}, \ldots, z_{k}\right) \in \mathbb{A}^{k}\right.$ $\left.\sum_{i=1}^{k} z_{i}^{2}=0\right\}$. Then we have, for $k \geq 1$,

$$
P\left(\mathscr{Y}_{k}\right)=\left\{\begin{array}{l}
q^{k-1} \text { if } k \text { is odd, } \\
q^{(k-1) / 2}+q^{k / 2}+q^{k-1} \quad \text { if } k \text { is even. }
\end{array}\right.
$$

(5.6) Proposition. Let

$$
\mathscr{V}(r, s)=\left\{\left[u_{1}, \ldots, u_{r}, z_{1}, \ldots, z_{s}\right] \in \mathbb{P}^{r+s-1} \mid \sum_{i=1}^{s} z_{i}^{2}=0\right\}
$$

(where $s \geq 0, r \geq 0$, but we exclude $r=s=0$ ). Then

(i)

for $s$ odd, $P(\mathscr{V}(r, s))=1+q+q^{2}+\cdots+q^{r+s-2}=P_{e}(\mathscr{V}(r, s))$,

(ii)

for $s$ even, $P(\mathscr{V}(r, s))=1+q+\cdots+q^{r+s-2}+q^{r+s / 2-1}=P_{e}(\mathscr{V}(r, s))$.

Proof. If $s$ is odd, we use induction on $r$, noting that $\mathscr{V}(0, s)=\mathscr{P}_{s-1}$, for which the result is true by (5.5). Moreover $\mathscr{V}(r, s)-\mathscr{V}(r-1, s) \simeq \mathbb{A}^{r-1} \times$ $\mathscr{Y}_{s}$ whose cohomology is given by (5.5), (5.2) and the Künneth formula. It is now routine to complete the proof using the cohomology exact sequence of the filtration $\mathscr{V}(r, s) \supset \mathscr{V}(r-1, s)$.

If $s$ is even, we may assume $s>0$, for if $s=0$ the result is trivial. Then use the filtration $\mathscr{V}(r, s) \supset \mathscr{V}(r, s-1)$ and the fact that $\mathscr{V}(r, s)-$ $\mathscr{V}(r, s-1) \simeq \mathbb{A}^{r} \times \mathscr{X}_{s-1}$, together with the result for odd $s$ (already proved).

(5.7) Corollary. For integers $a, b, s \geq 0$ with $(a, b, s) \neq(0,0,0)$, let $\mathscr{V}(a, b, s)$ be the variety $\left\{\left[v_{1}, \ldots, v_{2}, u_{1}, \ldots, u_{b}, z_{1}, \ldots, z_{s}\right] \in \mathbb{P}^{a+b+s-1}\right\}$ $\left.v_{1} v_{a}+v_{2} v_{a-1}+\cdots+\sum_{i=1}^{s} z_{i}^{2}=0\right\}$. Then

(i)

$$
\text { if } a+s \text { is odd, then } \begin{aligned}
P(\mathscr{V}(a, b, s)) & =1+q+\cdots+q^{a+b+s-2} \\
& =P_{e}(\mathscr{V}(a, b, s)),
\end{aligned}
$$

(ii)

$$
\begin{aligned}
& \text { if } a+s \text { is even, then } P(\mathscr{V}(a, b, s)) \\
& \quad=1+q+\cdots+q^{a+b+s-2}+q^{(a+s) / 2+b-1} \\
& \quad=P_{e}(\mathscr{V}(a, b, s)) .
\end{aligned}
$$

This is clear, since a linear automorphism of $\mathbb{P}^{a+b+s-2}$ takes $\mathscr{V}(a, b, s)$ into $\mathscr{V}(b, a+s)$ (in the notation of (5.6)). 
(5.8) Lemma. Let

$$
\begin{aligned}
& \mathscr{W}(d, e, s)=\left\{\left[u_{1}, \ldots, u_{d}, z_{1}, \ldots, z_{s}\right] \in \mathbb{P}^{d+s-1} \mid\right. \\
& \left.\qquad \sum_{i=1}^{s} z_{i}^{2}=0,\left(u_{1}, \ldots, u_{e}\right) \neq(0, \ldots, 0)\right\} \quad(e \leq d) .
\end{aligned}
$$

If $s$ is odd, then

$$
P(\mathscr{W}(d, e, s))=q^{D-e+1}+q^{D-e+2}+\cdots+q^{D}\left(=P_{e}(\mathscr{W}(d, e, s))\right)
$$

where $D=d+s-2=\operatorname{dim} \mathscr{W}(d, e, s)$ (as complex variety).

Proof. If $e=0$ then $\mathscr{W}(d, e, s)=\mathscr{V}(d, s)$ and the result follows from (5.6). If $e>1$, then $\mathscr{W}(d, e, s) \supset \mathscr{W}(d-1, e-1, s)$ (equation: $u_{1}=0$ ) and $\mathscr{W}(d, e, s)-\mathscr{W}(d-1, e-1, s) \simeq \mathbf{A}^{d-1} \times \mathscr{Y}_{s}$. Repeating this procedure, we get a filtration of $\mathscr{W}(d, e, s)$ whose final term is $\mathscr{W}(d-e+1,1, s) \simeq$ $\mathbb{A}^{d-e} \times \mathscr{Y}_{s}$. The result now follows by repeated application of Lemma 5.2(i).

When $s$ is even, the argument of (5.8) breaks down. However we shall prove

(5.9) Proposition. Let

$$
\begin{aligned}
\mathscr{W}=\mathscr{W}(d, e, s)=\left\{\left[u_{1}, \ldots, u_{d}, z_{1}, \ldots, z_{s}\right]\right. & \in \mathbb{P}^{d+s-1} \mid \sum_{i=1}^{s} z_{i}^{2}=0, \\
& \left.\left(u_{1}, \ldots, u_{e}\right) \neq(0, \ldots, 0)\right\} .
\end{aligned}
$$

If $s$ is even and non-zero, and $e \geq 1$, then (writing $D=d+s-2$ as above)

$$
P_{0}(\mathscr{W})=q^{D-e-s / 2+1}, \quad P_{e}(\mathscr{W})=q^{D-e+1}+q^{D-e+2}+\cdots+q^{D}+q^{D-(s-2) / 2}
$$

(that is $\left.P(w)=q^{D-e+1}+\cdots+q^{D}+q^{D-(s-1) / 2}+q^{D-e-(s-3) / 2)}\right)$.

Proof. Consider the filtration $\mathscr{W} \supset \mathscr{W}^{\prime}=\mathscr{W}(d, e, s-1)$ (equation: $\left.z_{s}=0\right)$. Then $\mathscr{W}-\mathscr{W}^{\prime} \cong\left(\mathbb{A}^{d}-\mathbb{A}^{d-e}\right) \times \chi_{s-1}$ and one easily sees that $H_{c}^{i}\left(\mathscr{W}-\mathscr{W}^{\prime}\right)$ vanishes except in degrees $2 D-s-2 e+3,2 D+1-2 e$, $2 D-s+2,2 D$ (where it is $\mathbb{C}$ ). Using (5.8), one obtains the exact sequence

$$
\begin{aligned}
0 \rightarrow H_{c}^{2 i}\left(\mathscr{W}-\mathscr{W}^{\prime}\right) \rightarrow H_{c}^{2 i}(\mathscr{W}) \rightarrow H_{c}^{2 i}\left(\mathscr{W}^{\prime}\right) \\
\rightarrow H_{c}^{2 i+1}\left(\mathscr{W}-\mathscr{W}^{\prime}\right) \rightarrow H_{c}^{2 i+1}(\mathscr{W}) \rightarrow 0 .
\end{aligned}
$$


After a bookkeeping exercise in dimensions, one obtains the result that either (5.9) holds, or else

$$
P_{o}(\mathscr{W})=q^{D-e-s / 2+1}+q^{D-e}
$$

and

$$
P_{e}(\mathscr{W})=q^{D-e+1}+\cdots+q^{D}+q^{D-(s-2) / 2}+q^{D-e} .
$$

To resolve the ambiguity, we consider

$$
\mathscr{W}=\mathscr{W}(d, e, s) \hookrightarrow \mathscr{W}(d, e, s+1)=\widetilde{\mathscr{W}},
$$

and the corresponding exact sequence; here $H_{c}^{*}(\widetilde{\mathscr{W}})$ is known (by (5.8)) and we have

$$
\begin{array}{r}
0 \rightarrow H_{c}^{2 i-1}(\mathscr{W}) \rightarrow H_{c}^{2 i}(\widetilde{\mathscr{W}}-\mathscr{W}) \rightarrow H_{c}^{2 i}(\widetilde{\mathscr{W}}) \\
\rightarrow H_{c}^{2 i}(\mathscr{W}) \rightarrow H_{c}^{2 i+1}(\widetilde{\mathscr{W}}-\mathscr{W}) \rightarrow 0
\end{array}
$$

where $\widetilde{\mathscr{W}}-\mathscr{W} \simeq\left(\mathbb{A}^{d}-\mathbb{A}^{d-e}\right) \times \mathscr{Z}_{s}$.

Putting $i=D-e$ in the above sequence and using the information above gives, after collecting terms, $H_{c}^{2(D-e)}(\mathscr{W})=0$ if $e \neq(s-2) / 2$. If $e=$ $(s-2) / 2$ one again enumerates the possibilities for non-zero terms in the sequence above, finally concluding that $H_{c}^{2(D-e)}(\mathscr{W})=\mathbb{C}$ in this case, as stated in (5.9).

We summarise the previous two results in

(5.10) THEOREM. Let

$$
\begin{aligned}
\mathscr{W}=\mathscr{W}(d, e, s)=\left\{\left[u_{1}, \ldots, u_{d}, z_{1}, \ldots, z_{s}\right] \in \mathbb{P}^{d+s-1} \mid \sum_{i=1}^{s} z_{i}^{2}=0\right. \\
\text { and } \left.\left(u_{1}, \ldots, u_{e}\right) \neq(0,0, \ldots, 0)\right\} .
\end{aligned}
$$

Assume that $1 \leq e \leq d$ (if $e=0$ cf. (5.6)). Then (writing $D=d+s-2=$ $\operatorname{dim} \mathscr{W})$ we have

(i) if $s$ is odd, $P_{e}(\mathscr{W})=q^{D-e+1}+q^{D-e+2}+\cdots+q^{D}$ and $P_{0}(\mathscr{W})=0$,

(ii) if $s$ is even and $\neq 0, P_{e}(\mathscr{W})=q^{D-e+1}+\cdots+q^{D}+q^{D-(s-2) / 2}$ and $P_{o}(\mathscr{W})=q^{D-e-(s-2) / 2}$,

(iii) if $s=0$, then (i) holds, with $D=\operatorname{dim} \mathscr{W}=d+s-1=d-1$. 
Finally, we need to investigate the varieties

$$
\begin{aligned}
& \mathscr{W}(a, b, e, s)=\left\{\left[v_{1}, \ldots, v_{a}, v_{a+1}, \ldots, v_{a+b}, z_{1}, \ldots, z_{s}\right]\right. \\
&\left.\in \mathscr{V}(a, b, s) \mid\left(v_{1}, \ldots, v_{e}\right) \neq(0, \ldots, 0)\right\}
\end{aligned}
$$

(see (5.7)) where

$$
\begin{aligned}
& \mathscr{V}(a, b, s)=\left\{\left[v_{1}, \ldots, v_{a}, v_{a+1}, \ldots, v_{a+b}, z_{1}, \ldots, z_{s}\right]\right. \\
&\left.\in \mathbb{P}^{a+b+s-1} \mid v_{1} v_{a}+v_{2} v_{a-1}+\cdots+z_{1}^{2}+\cdots+z_{s}^{2}=0\right\}
\end{aligned}
$$

and we assume $s \geq 0,0 \leq e \leq a+b$.

First observe that for $e=0, \mathscr{W}(a, b, e, s)=\mathscr{V}(a, b, s)$, whose cohomology has already been described. Also, if $a=0$ then

$$
\mathscr{W}(0, b, e, s)=\mathscr{W}(b, e, s)
$$

which is treated in (5.10) above. Moreover if $e=1, \mathscr{W}(a, b, e, s)$ is easily recognised: we have

$$
\mathscr{W}(1, b, 1, s) \simeq \mathbb{A}^{b} \times \mathscr{X}_{s},
$$

while

$$
\mathscr{W}(a, b, 1, s) \simeq \mathbb{A}^{a+b+s-2} \quad(a>1) .
$$

Thus we may suppose $e>1, a \geq 1$ and reduce to the case $e=1$.

(5.11) LemmA. Suppose $1<e \leq 1+a / 2$. Then $\mathscr{W}(a, b, e, s)$ has a filtration with successive strata given by $\mathbb{A}^{D}, \mathbf{A}^{D-1}, \ldots, \mathbf{A}^{D-(e-2)}, \mathscr{W}(a-2 e+2$, $b+e-1,1, s)$ (where $D=a+b+s-2=\operatorname{dim} \mathscr{W}(a, b, e, s)$ as complex variety).

Proof. Let $\mathscr{W}^{\prime}=\left\{x \in \mathscr{W}=\mathscr{W}(a, b, e, s) \mid v_{1}=0\right\}$. Then clearly $\mathscr{W}^{\prime} \simeq \mathscr{W}(a-2, b+1, e-1, s)$ and $\mathscr{W}-\mathscr{W}^{\prime} \simeq \mathbb{A}^{D}$. Moreover if $e-1>1$, then we still have $1<e-1 \leq 1+(a-2) / 2$, so that we may repeat the above procedure, obtaining $\mathscr{W}^{\prime \prime} \simeq \mathscr{W}(a-4, b+2, e-2, s\}$ and $\mathscr{W}^{\prime}-\mathscr{W}^{\prime \prime} \simeq$ $\mathrm{A}^{D-1}$. The assumption on $e$ proves that this procedure terminates with $\mathscr{W}(a-2 e+2, b+e-1,1, s)$ as stated.

(5.12) Corollary. If $1<e \leq a / 2+1$, then

$$
H_{c}^{i}(\mathscr{W}(a, b, e, s))=\left\{\begin{array}{l}
\mathbb{C} \text { if } i=2 j, D \geq j \geq D-(e-2), \\
H_{c}^{i}(\mathscr{W}(a-2 e+2, b+e-1,1, s)) \\
\quad \text { if } i \leq 2(D-(e-1)), \\
0 \text { otherwise. }
\end{array}\right.
$$

This follows from repeated application of Lemma (5.2)(i) to the filtration of (5.11). 
Now if $a \geq 2$, then $\mathscr{W}(a, b, 1, s) \simeq \mathbb{A}^{a+b+s-2}$. Hence as an easy consequence of $(5.12)$, we have

(5.13) COROLlary. If $2 e \leq a$, then

$$
P_{e}(\mathscr{W}(a, b, e, s))=q^{D-e+1}+q^{D-e+2}+\cdots+q^{D}
$$

and

$$
P_{o}(\mathscr{W}(a, b, e, s))=0
$$

For in this case, $a-2 e+2 \geq 2$.

Hence we are reduced to a consideration of the case $2 e>a$.

(5.14) Lemma. Suppose $a$ is even and that $2 e>a$. Then

$$
H_{c}^{i}(\mathscr{W}(a, b, e, s))=\left\{\begin{array}{l}
\mathbb{C} \text { if } i=2 j, D \geq j \geq D-a / 2+1, \\
H_{c}^{i}(\mathscr{W}(b+a / 2, e-a / 2, s)) \text { if } i \leq 2 D-a, \\
0 \text { otherwise. }
\end{array}\right.
$$

Proof. Using the same construction as for Corollary (5.12), we see that $\mathscr{W}(a, b, e, s)$ has a filtration whose successive strata are

$$
\mathbb{A}^{D}, \mathbb{A}^{D-1}, \ldots, \mathbb{A}^{D-\frac{a}{2}+1}, \mathscr{W}\left(0, b+\frac{a}{2}, e-\frac{a}{2}, s\right)=\mathscr{W}\left(b+\frac{a}{2}, e-\frac{a}{2}, s\right)
$$

in the notation of (5.10). The result now again follows by applying (5.2)(i) repeatedly.

In view of (5.10), this leaves only the case where $a$ is odd and $2 e>a$. For this case, we have, corresponding to (5.14), and with a similar proof,

(5.15) LeMMA. If $a$ is odd and $2 e>a$ then

$H_{c}^{i}(\mathscr{W}(a, b, e, s))=\left\{\begin{array}{l}\mathbb{C} \text { if } i=2 j, D \geq j \geq D-\frac{a-1}{2}+1, \\ H_{c}^{i}\left(\mathscr{W}\left(1, b+\frac{a-1}{2}, e-\frac{a-1}{2}, s\right)\right) \quad \text { if } i \leq 2 D-a+1, \\ 0 \text { otherwise }\end{array}\right.$ (where $D=a+b+s-2$ ).

Thus we are reduced to the case $a=1$ (and $e>1$ ). For the remainder of this section, write $\mathscr{W}=\mathscr{W}(1, b, e, s)$.

(5.16) Lemma. Suppose $s$ is odd, $e>1$. Then

(i) $P_{0}(\mathscr{W})=0$, 
(ii) $P_{e}(\mathscr{W})=q^{D-e+1}+q^{D-e+2}+\cdots+q^{D}+q^{D-(s-1) / 2}$, where $D=\operatorname{dim} \mathscr{W}=(b+1+s)-2=b+s-1$.

Proof. Let $\mathscr{W}^{\prime}=\mathscr{W}(b, e-1, s) \subset \mathscr{W}$ (equation: $\left.u_{1}=0\right)$. Then $\mathscr{W}-\mathscr{W}^{\prime} \simeq \mathbb{A}^{b} \times \mathscr{E}_{s}$, and so $H_{c}^{i}\left(\mathscr{W}-\mathscr{W}^{\prime}\right)=\mathbb{C}$ if $i=2 D$ or $2 D-s+1$ and zero otherwise. Since $H_{c}^{i}\left(\mathscr{W}^{\prime}\right)=0$ for $i$ odd (see (5.10)), it follows from the cohomology exact sequence of the inclusion $\mathscr{W}^{\prime} \hookrightarrow \mathscr{W}$ that $P_{o}(\mathscr{W})=0$.

Using this, another straightforward application of the cohomology exact sequence gives the formula for $P_{e}(\mathscr{W})$.

We treat the final case in

(5.17) LemMa. With notation as in (5.16) suppose $s$ is even, $s>0$ and $e>1$. Then

(i) $P_{o}(\mathscr{W})=q^{D-(s-2) / 2-e}$,

(ii) $P_{e}(\mathscr{W})=q^{D-e+1}+\cdots+q^{D}$.

Proof. Let $\mathscr{W}_{1}=\left\{\left[u_{1}, u_{2}, \ldots, u_{b+1}, z_{1}, \ldots, z_{s}\right] \in \mathscr{W} \mid u_{2}=0\right\}$. Then $\mathscr{W}_{1} \simeq \mathscr{W}(1, b-1, e-1, s)$ and $\mathscr{W}-\mathscr{W}_{1} \simeq \mathbb{A}^{b-1} \times \mathscr{Y}_{s+1}$. Hence $H_{c}^{i}\left(\mathscr{W}-\mathscr{W}_{1}\right)=\mathbb{C}$ if $i=2 D$ and 0 otherwise, since $s$ is even. Repeating this procedure and applying (5.2)(i) repeatedly, we obtain

$$
H_{c}^{i}(\mathscr{W})=\left\{\begin{array}{l}
\mathbb{C} \text { if } i=2 j, D \geq j \geq D-e+2, \\
H_{c}^{i}(\mathscr{W}(1, b-e+1,1, s)) \text { if } i \leq 2(D-e+1), \\
0 \text { otherwise. }
\end{array}\right.
$$

Moreover $\mathscr{W}(1, b-e+1,1, s) \simeq \mathbb{A}^{b-e+1} \times \chi_{s}$, whose cohomology is given by (5.2) and (5.3). Collecting these facts, we obtain (5.17).

We collect the results of (5.11) to (5.17) in

(5.18) THEOREM. For integers $a, b, e, s \geq 0$, define the variety

$$
\mathscr{W}(a, b, e, s)=\mathscr{W}
$$

by

$$
\begin{aligned}
& \mathscr{W}=\left\{\left[u_{1}, \ldots, u_{a}, u_{a+1}, \ldots, u_{a+b}, z_{1}, \ldots, z_{s}\right]\right. \\
& \in \mathbb{P}^{a+b+s-1} \mid u_{1} u_{a}+u_{2} u_{a-1}+\cdots+z_{1}^{2}+\cdots+z_{s}^{2} \\
& \left.=0,\left(u_{1}, \ldots, u_{e}\right) \neq(0, \ldots, 0)\right\} \quad(e \leq a+b) .
\end{aligned}
$$

Write $D=\operatorname{dim}_{\mathbb{C}} \mathscr{W}=a+b+s-2$ and $P_{o}(\mathscr{W})=\sum_{i=0}^{\infty} \operatorname{dim} H_{c}^{2 i+1}(\mathscr{W}) q^{i}$, and $P_{e}(\mathscr{W})=\sum_{i=0}^{\infty} \operatorname{dim} H_{c}^{2 i}(\mathscr{W}) q^{i}$. Then the values of $P_{o}$ and $P_{e}$ are given in the following table. 


\begin{tabular}{|l|c|c|}
\hline & $P_{o}(\mathscr{W})$ & $P_{e}(\mathscr{W})$ \\
\hline $2 e \leq a$ & 0 & $q^{D-e+1}+q^{D-e+2}+\cdots+q^{D}$ \\
\hline $\begin{array}{l}2 e>a \\
a \text { even } \\
s \text { even, }>0\end{array}$ & $q^{D-e-\frac{s-2}{2}}$ & $q^{D-e+1}+q^{D-e+2}+\cdots+q^{D}+q^{D-\frac{a}{2}-\frac{s-2}{2}}$ \\
\hline $\begin{array}{l}2 e>a \\
a \text { odd } \\
s \text { odd }\end{array}$ & 0 & $q^{d-e+1}+q^{D-e+2}+\cdots+q^{D}+q^{D-\frac{s-1}{2}-\frac{a-1}{2}}$ \\
\hline $\begin{array}{l}2 e>a \\
a \text { odd } \\
s \text { even, }>0\end{array}$ & $q^{D-e-\frac{s-2}{2}}$ & $q^{D-e+1}+q^{D-e+2}+\cdots+q^{D}$ \\
\hline $\begin{array}{l}2 e>a \\
a \text { even } \\
s \text { odd or } 0\end{array}$ & 0 & $q^{D-e+1}+q^{D-e+2}+\cdots+q^{D}$ \\
\hline
\end{tabular}

\section{The linear and symplectic groups}

The proof of Theorem 2.4 for the linear and symplectic groups involves an easy application of the inequality (4.8).

\section{The linear case.}

Here we suppose $G$ is of type $A_{n-1}\left(G=G L_{n}\right.$ or $\left.S L_{n} \ldots\right)$; the nilpotent orbits in $\mathscr{G}$ correspond to ordered partitions $\lambda$ of $n$. All nilpotent orbits are of parabolic type. Take $P$ to be a parabolic subgroup corresponding to a subsystem of type $A_{n-2}$. Then $\operatorname{Ind}_{W(L)}^{W}(1)=1+\rho$ and $\mathscr{P}=\mathbb{P}^{n-1}(\mathbb{C})$.

Now take $A$ of type $\lambda=\left(\lambda_{1} \geq \lambda_{2} \geq \cdots \geq \lambda_{p}>0\right)$. Then $\mathscr{P}_{A}=\mathbb{P}(\operatorname{ker} A)=$ $\mathbb{P}^{p-1}$ and $\mathscr{P}-\mathscr{P}_{A}=\mathbb{P}^{n-1}-\mathbb{P}^{p-1}$. Moreover $\left\langle Q_{0}, \rho\right\rangle=q+q^{2}+\cdots+q^{n-1}$ (see (2.5)). From (5.2), it follows that (4.8) reduces (with $\chi=\rho$ ) in this 
case to

$$
\begin{aligned}
\sup & \left\{0, q+q^{2}+\cdots+q^{n-1}-\left(q^{p}+q^{p+1}+\cdots+q^{n-1}\right)\right\} \leq\left\langle Q_{A}, \rho\right\rangle \\
& \leq \inf \left\{1+q+\cdots+q^{p-1}, q+q^{2}+\cdots+q^{n-1}\right\}
\end{aligned}
$$

from which it follows immediately that

$$
\left\langle Q_{A}, \rho\right\rangle=q+q^{2}+\cdots+q^{p-1} .
$$

Comparison with (2.9) completes the proof of (2.4) for type $A$.

The symplectic case (type $C_{n}$ ).

Suppose $G=\operatorname{Sp}(2 n, \mathbb{C}$ ) and take $P$ (as in Section 3 ) to be the isotropy subgroup of a line in the symplectic space $\mathbb{C}^{2 n}$. Then $\mathscr{P}=G / P=\mathbb{P}^{2 n-1}$, $W(L)$ is a parabolic subgroup of type $C_{n-1}$ and $\operatorname{Ind}_{W(L)}^{W}(1)=1+\rho+\xi$, where $\rho$ is the reflection representation and $\xi$ is irreducible of degree $n-1$ (it is the reflection representation of the symmetric group $S_{n}$, lifted to $W\left(C_{n}\right)$ via the canonical surjection $\left.W\left(C_{n}\right) \rightarrow S_{n}\right)$.

The nilpotent orbits in $\mathscr{G}$ are given (cf. [27]) by partitions $\lambda$ of $2 n$, such that any odd part occurs an even number of times. Suppose $J$ is a subset of $\Pi$ which corresponds to a subsystem of type $A_{i_{1}} \times A_{i_{2}} \times \cdots \times A_{i_{t}} \times C_{k}$. The corresponding partition (written in unordered form) is $\left(i_{1}+1, i_{1}+1, \ldots, i_{t}+\right.$ $\left.1, i_{t}+1,2 k, 1^{s}\right)$ where $\sum_{j=1}^{t} 2\left(i_{j}+1\right)+2 k+s=2 n \quad(k=0$ corresponds to the case where there is no component of type $C$ ). Thus the number $p$ of parts of $\lambda$ is given by

$$
p= \begin{cases}2 t+s+1 & \text { if } J \text { has a component of type } C, \\ 2 t+s & \text { if } J \text { has no component of type } C,\end{cases}
$$

and for comparison with (2.9) it is useful to note that $2 t+s=2(n-|J|)$.

Again from (2.5) we have in this case

$$
\left\langle Q_{0}, \rho\right\rangle=q+q^{3}+\cdots+q^{2 n-1} .
$$

Moreover since $\left\langle Q_{0}, 1\right\rangle=1$ and by $(4.3)(\mathrm{i})$ we have $\operatorname{dim}\left(Q_{0}^{W(L)}\right)=$ $\left\langle Q_{0}, 1+\xi+\rho\right\rangle\left(=P_{e}(\mathscr{P})\right.$ by Borho and MacPherson $)$, we have

$$
\left\langle Q_{0}, \xi\right\rangle=q^{2}+q^{4}+\cdots+q^{2 n}
$$

Now take $A$ nilpotent, with corresponding partition $\lambda$. Write $p$ for the number of parts of $\lambda$ and $s$ for the multiplicity of 1 in $\lambda$. Then with the above notation we have $\mathscr{P}_{A}=\mathbb{P}(\operatorname{ker} A)=\mathbb{P}^{p-1}$ and $\mathscr{P}-\mathscr{P}_{A}=\mathbb{P}^{2 n-1}-\mathbb{P}^{p-1}$. Using (as above) the relation $\operatorname{dim}\left(Q_{A}^{W(L)}\right)=\left\langle Q_{A}, 1+\xi+\rho\right\rangle=P_{e}\left(\mathscr{P}_{A}\right)$, we obtain (using the fact that $\left\langle Q_{A}, 1\right\rangle=1$ for all $A$ )

$$
\left\langle Q_{A}, \rho\right\rangle+\left\langle Q_{A}, \xi\right\rangle=q+q^{2}+\cdots+q^{p-1}
$$


Next, apply (4.8) to $\rho$ and to $\xi$ : we have

$$
\begin{aligned}
\sup & \left\{0, q+q^{3}+\cdots+q^{2 n-1}-\left(q^{p}+\cdots+q^{2 n-1}\right)\right\} \\
& \leq\left\langle Q_{A}, \rho\right\rangle \leq \inf \left\{1+q+\cdots+q^{p-1}, q+q^{3}+\cdots+q^{2 n-1}\right\}
\end{aligned}
$$

whence $\left\langle Q_{A}, \rho\right\rangle \leq q+q^{3}+\cdots+q^{p-1}$ (only odd powers occurring). Similarly, $\left\langle Q_{A}, \xi\right\rangle \leq q^{2}+q^{4}+\cdots+q^{p-1}$ (only even powers occurring).

It follows from (6.4) that

$$
\left\langle Q_{A}, \rho\right\rangle= \begin{cases}q+q^{3}+\cdots+q^{p-1} & \text { if } p \text { is even, } \\ q+q^{3}+\cdots+q^{p-2} & \text { if } p \text { is odd }\end{cases}
$$

and that

$$
\left\langle Q_{A}, \xi\right\rangle= \begin{cases}q^{2}+q^{4}+\cdots+q^{p-2} & \text { if } p \text { is even, } \\ q^{2}+q^{4}+\cdots+q^{p-1} & \text { if } p \text { is odd } .\end{cases}
$$

Combining (6.5) with (6.1) and comparing with (2.9) completes the proof of (2.4) for the symplectic case. (One obtains, in the notation of (6.1), that

$$
\left\langle Q_{A}, \rho\right\rangle=q+q^{3}+\cdots+q^{2 t+s-1} \text { and } 2 t+s-1=2(n-|J|-1) \text {.) }
$$

\section{The orthogonal groups}

Case 1. The group $S O(2 n+1)$ (type $\left.B_{n}\right)$

The nilpotent orbits in $\mathscr{G}$ correspond to partitions $\lambda=\left(\lambda_{1} \geq \lambda_{2} \geq \cdots \geq\right.$ $\lambda_{p}>0$ ) of $2 n+1$ such that any even part occurs an even number of times (see [27, page 259]). For a nilpotent element $A$ corresponding to the partition $\lambda$ we write

$$
\begin{gathered}
s=\#\left\{i \mid \lambda_{i}=1\right\}, \quad p=\text { number of parts of } \lambda \text { (always odd here), } \\
\quad r=p-s, \quad N=2 n+1-2 .
\end{gathered}
$$

Take $P$ to be the maximal parabolic subgroup of $G$ which is the isotropy group of an isotropic line in the orthogonal space $\mathbb{C}^{2 n+1}$ on which $G$ acts naturally. It is shown in [27] that given $A$, one can choose a basis of $\mathbb{C}^{2 n+1}$ with respect to which the quadratic from $Q$ which is left invariant by $G$ has the form

$$
Q\left(x_{1}, \ldots, x_{N}, z_{1}, \ldots, z_{s}\right)=x_{1} x_{N}+x_{2} x_{N-1}+\cdots+z_{1}^{2}+z_{2}^{2}+\cdots+z_{s}^{2}
$$

and the spaces $\mathscr{P}=G / P$ and $\mathscr{P}_{A}=\{g P \in \mathscr{P} \mid A \in \operatorname{Adg}$ Lie $P\}$ are given by

$$
\left\{\begin{array}{l}
\mathscr{P}=\left\{\left[x_{1}, \ldots, x_{N}, z_{1}, \ldots, z_{s}\right]=x \in \mathbb{P}^{2 n} \mid Q(x)=0\right\}, \\
\mathscr{P}=\left\{x \in \mathscr{P} \mid x_{1}=x_{2}=\cdots=x_{N-r}=0\right\} .
\end{array}\right.
$$


Suppose $J$ is a subset of $\Pi$ which corresponds to a subsystem of type $A_{i_{1}} \times A_{i_{2}} \times \cdots \times A_{i_{i}} \times B_{k}$. The corresponding partition is $\left(i_{1}+1, i_{1}+1, \ldots, i_{t}+\right.$ $\left.1, i_{t}+1,2 k+1,1^{s}\right)$ where $\sum_{j=1}^{t} 2\left(i_{j}+1\right)+2 k+1+s=2 n+1$ (if there is a component of type $B_{k}$ ). In this case it is easily verified that $2 t+s=$ $2(n-|J|)=p-1$. Similarly if there is no component of type $B_{k}, 2(n-|J|)$ $=2 t+s-1=p-1$. Thus in analogy with (6.1), we have here

(7.3) if $\lambda$ is of parabolic type (corresponding to $J \subseteq \Pi$ ) then

$$
2(n-|J|)=p-1=\left\{\begin{array}{l}
2 t+s \text { if } J \text { has a component of type } B, \\
2 t+s-1 \text { if } J \text { has no component of type } B .
\end{array}\right.
$$

Now let $A$ be an arbitrary nilpotent element in $\mathscr{G}$ with corresponding partition $\lambda$. From (7.2) it is clear that (in the notation of (5.7), (5.6) and (5.18)) we have

$$
\begin{aligned}
& \text { (i) } \mathscr{P} \simeq \mathscr{V}(N, 0, s), \text { (ii) } \mathscr{P}_{A} \simeq \mathscr{V}(r, s), \\
& \text { (iii) } \mathscr{P}-\mathscr{P}_{A} \simeq \mathscr{W}(N, 0, N-r, s) .
\end{aligned}
$$

Here (ii) follows from the fact that since each of the $r$ parts not equal to 1 has cardinality at least 2 , we have $r \leq N / 2$.

Again as in the case of type $C, \operatorname{Ind}_{W(L)}^{W}(1)=1+\rho+\xi$, where $\rho$ and $\xi$ are as in Section 6 above, and we have

$$
\left\langle Q_{0}, \rho\right\rangle=q+q^{3}+\cdots+q^{2 n-1} .
$$

Now $N+s=2 n+1$, which is odd. Hence by (5.7) we have

$$
P_{e}(\mathscr{P})=P_{e}(\mathscr{V}(N, 0, s))=1+q+\cdots+q^{2 n-1} .
$$

Thus using (4.3)(i), we deduce

$$
\left\langle Q_{0}, \xi\right\rangle=q^{2}+q^{4}+\cdots+q^{2 n-2} .
$$

Similarly it follows from (5.6) and (7.4)(ii) that

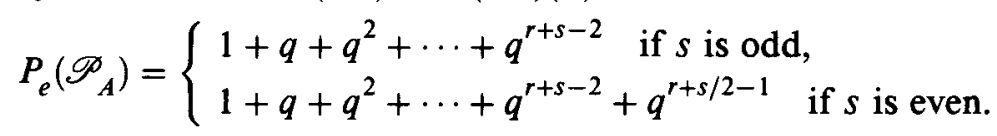

Moreover from (5.18) and (7.4)(iii) we have

$$
P_{o}\left(\mathscr{P}-\mathscr{P}_{A}\right)= \begin{cases}0 & \text { if } s \text { is odd or } 0, \\ q^{r+s / 2-1} & \text { if } s \text { is even and }>0,\end{cases}
$$

and

$$
P_{e}\left(\mathscr{P}-\mathscr{P}_{A}\right)=q^{p-1}+q^{p}+\cdots+q^{2 n-1} .
$$

In an analogous fashion to (6.4) we deduce from (7.8) that (7.11) $\left\langle Q_{A}, \rho\right\rangle+\left\langle Q_{A}, \xi\right\rangle=\left\{\begin{array}{l}q+q^{2}+\cdots+q^{r+s-2} \text { if } s \text { is odd, } \\ q+q^{2}+\cdots+q^{r+s-2}+q^{r+s / 2-1} \text { if } s \text { is even. }\end{array}\right.$ 
Now apply (4.8) to $\rho$ and $\xi$ respectively; we obtain

$$
\begin{aligned}
\sup \{0 & \left., q+q^{3}+\cdots+q^{2 n-1}-\left(q^{p-1}+q^{p}+\cdots+q^{2 n-1}\right)\right\} \leq\left\langle Q_{A}, \rho\right\rangle \\
\leq & \left\{\begin{array}{r}
\inf \left\{1+q+\cdots+q^{r+s-2}, q+q^{3}+\cdots+q^{2 n-1}\right\} \quad \text { if } s \text { is odd } \\
\inf \left\{1+q+\cdots+q^{r+s-2}+q^{r+s / 2-1}, q+q^{3}+\cdots+q^{2 n-1}+q^{r+s / 2-1}\right\} \\
\text { if } s \text { is even and }>0 \\
\inf \left\{1+q+\cdots+q^{r-1}, q+q^{3}+\cdots+q^{2 n-1}+q^{r-1}\right\} \quad \text { if } s=0,
\end{array}\right.
\end{aligned}
$$

that is,

$$
\begin{aligned}
& q+q^{3}+\cdots+q^{p-2} \\
& \quad \leq\left\langle Q_{A}, \rho\right\rangle \leq\left\{\begin{array}{l}
q+q^{3}+\cdots+q^{p-2} \text { if } s \text { is odd } \\
q+q^{3}+\cdots+q^{p-2}+q^{r+s / 2-1} \text { if } s \text { is even. }
\end{array}\right.
\end{aligned}
$$

Similarly for $\xi$ we have

$$
\begin{aligned}
q^{2}+ & q^{4}+\cdots+q^{p-3} \\
& \leq\left\langle Q_{A}, \xi\right\rangle \leq\left\{\begin{array}{l}
q^{2}+q^{4}+\cdots+q^{p-3} \text { if } s \text { is odd } \\
q^{2}+q^{4}+\cdots+q^{p-3}+q^{r+s / 2-1} \text { if } s \text { is even. }
\end{array}\right.
\end{aligned}
$$

The inequalities (7.12) and (7.13) are valid for all $A$. To resolve the ambiguity when $s$ is even in the parabolic case, apply (4.9): if $A$ is parabolic of type $J \subseteq \Pi$, then using (7.3) we have

$$
\left\langle Q_{A}, \rho\right\rangle_{q=1}=\left\langle\operatorname{Ind}_{W_{J}}^{W}(1), \rho\right\rangle=n-|J|=\frac{p-1}{2} .
$$

Comparing with (7.12) we obtain

(7.15) if $A \in \mathscr{G}$ is a nilpotent element of parabolic type and the corresponding partition $\lambda$ has $p$ parts, of which $s$ are equal to 1 , then

$$
\left\langle Q_{A}, \rho\right\rangle=q+q^{3}+\cdots+q^{p-2}
$$

and

$$
\left\langle Q_{A}, \xi\right\rangle=\left\{\begin{array}{l}
q^{2}+q^{4}+\cdots+q^{p-3} \text { if } s \text { is odd } \\
q^{2}+q^{4}+\cdots+q^{p-3}+q^{p-s / 2-1}
\end{array} \text { if } s\right. \text { is even. }
$$

(Note that $s$ is even if and only if $J$ has a component of type $B_{k}$.)

Comparing (7.15) with (2.9) (taking into account that $p=2(n-|J|)+1$ by (7.3)) completes the proof of (2.4) for the case of type $B_{n}$.

Finally, observe that (7.12) and (7.13) determine $\left\langle Q_{A}, \rho\right\rangle$ and $\left\langle Q_{A}, \xi\right\rangle$ completely for $s$ odd in the general (that is, not necessarily parabolic) case while for $s$ even there remains an ambiguity concerning the term $q^{r+s / 2-1}$ (cf. (7.11)). Below we indicate how an "Ennola-type" duality argument can resolve this ambiguity. 
CASE 2. The group $S O(2 n)$ (type $D_{n}$ ).

Here the nilpotent orbits in $\mathscr{G}$ correspond to partitions $\lambda=\left(\lambda_{1} \geq \cdots \geq\right.$ $\left.\lambda_{p}>0\right)$ of $2 n$ such that any even part occurs an even number of times. Define $s, p$ and $r$ as at the beginning of this section and write $N=2 n-s$. Choose $P$ in the same way as in Case 1 ; then as above there is a basis of $\mathbb{C}^{2 n}$ with respect to which the quadratic form $Q$ which is left invariant by $G$ has the form

$$
Q\left(x_{1}, \ldots, x_{N}, z_{1}, \ldots, z_{s}\right)=x_{1} x_{N}+x_{2} x_{N-1}+\cdots+z_{1}^{2}+\cdots+z_{s}^{2}
$$

and here the spaces $\mathscr{P}=G / P$ and $\mathscr{P}_{A}$ are given by

$$
\left\{\begin{array}{l}
\mathscr{P}=\left\{\left[x_{1}, \ldots, x_{N}, z_{1}, \ldots, z_{s}\right]=x \in \mathbb{P}^{2 n-1} \mid Q(x)=0\right\}, \\
\mathscr{P}_{A}=\left\{x \in \mathscr{P} \mid x_{1}=x_{2}=\cdots=x_{N-r}=0\right\}(r \leq N / 2) .
\end{array}\right.
$$

Thus in the notation of Section 5 we have again (as in (7.4))

$$
\mathscr{P} \simeq \mathscr{V}(N, 0, s), \quad \mathscr{P}_{A} \simeq \mathscr{V}(r, s), \quad \mathscr{P}-\mathscr{P}_{A} \simeq \mathscr{W}(N, 0, N-r, s) .
$$

Note that $p=r+s$ is even in this case.

As above we have $\operatorname{Ind}_{W(L)}^{W}(1)=1+\rho+\xi$, but

$$
\left\langle Q_{0}, \rho\right\rangle=q+q^{3}+\cdots+q^{2 n-3}+q^{n-1} .
$$

Due to the different parities of the integers concerned, the cohomology spaces are different. Since $N+s(=2 n)$ is even, we have by (5.7) that

$$
P_{e}(\mathscr{P})=1+q+\cdots+q^{2 n-2}+q^{n-1} .
$$

Thus, combining (7.19) and (7.20) we have

$$
\left\langle Q_{0}, \xi\right\rangle=q^{2}+q^{4}+\cdots+q^{2 n-2} .
$$

As above, we have from (5.6)

$$
P_{e}\left(\mathscr{P}_{A}\right)=\left\{\begin{array}{l}
1+q+q^{2}+\cdots+q^{r+s-2} \text { if } s \text { is odd or zero, } \\
1+q+q^{2}+\cdots+q^{r+s-2}+q^{r+s / 2-1} \text { if } s \text { is even, }
\end{array}\right.
$$

and from (5.18) we have

$$
P_{o}\left(\mathscr{P}-\mathscr{P}_{A}\right)=\left\{\begin{array}{l}
0 \text { if } s \text { is odd or zero, } \\
q^{r+s / 2-1} \text { if } s \text { is even and }>0,
\end{array}\right.
$$

and

$$
P_{e}\left(\mathscr{P}-\mathscr{P}_{A}\right)=q^{p-1}+q^{p}+\cdots+q^{2 n-2}+q^{n-1} .
$$

Moreover the equation (7.11) remains valid in the case under consideration (in view of (7.22)). 
As in the above case we apply (4.8), obtaining

$$
q+q^{3}+\cdots+q^{p-3} \leq\left\langle Q_{A}, \rho\right\rangle \leq\left\{\begin{array}{l}
q+q^{3}+\cdots+q^{p-3} \text { if } s \text { is odd, } \\
q+q^{3}+\cdots+q^{p-3}+q^{r+s / 2-1} \quad \text { if } s \text { is even, }
\end{array}\right.
$$

unless $n$ is odd and $n<p$ in which case we have

$$
q+q^{3}+\cdots+q^{p-3} \leq\left\langle Q_{A}, \rho\right\rangle \leq\left\{\begin{array}{rr}
q+q^{3}+\cdots+q^{p-3}+q^{n-1} & \text { if } s \text { is odd } \\
q+q^{3}+\cdots+q^{p-3}+q^{n-1}+q^{r+s / 2-1} & \\
\text { if } s \text { is even. }
\end{array}\right.
$$

Also we have

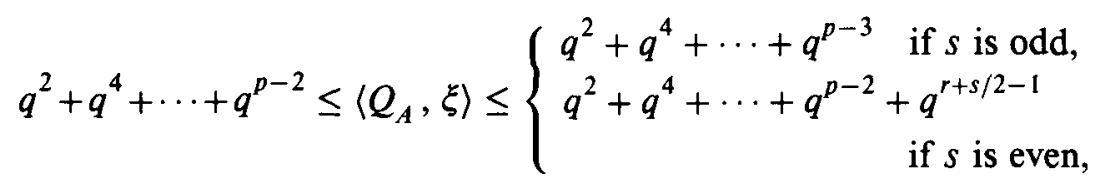

unless $n$ is odd and $n<p$ in which case we have

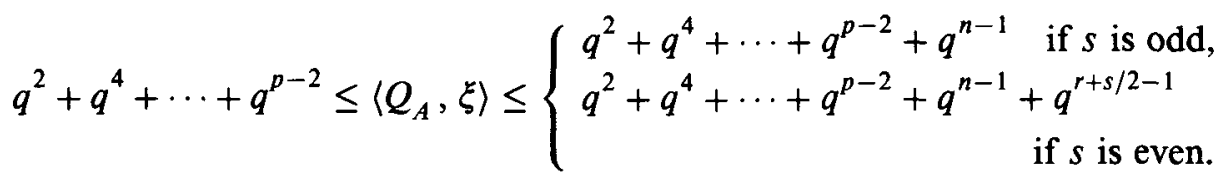

For the moment, exclude the case where $n$ is odd and $n<p$. Then

(7.26) if $s$ is odd, $\left\langle Q_{A}, \rho\right\rangle=q+q^{3}+\cdots+q^{p-3}$ and $\left\langle Q_{A}, \xi\right\rangle=q^{2}+q^{4}+$ $\cdots+q^{p-2}$.

To resolve the ambiguity here when $s$ is even, we apply (4.9) to the parabolic case. (Below we sketch how to apply the "Ennola-type" duality method to the case where $n$ is even to determine $\left\langle Q_{A}, \rho\right\rangle$ and $\left\langle Q_{A}, \xi\right\rangle$ for $s$ even in general.)

Suppose $A$ is of type $J$, a parabolic subsystem of $D_{n}$ of type $A_{i_{1}} \times A_{i_{2}} \times$ $\cdots \times A_{i_{t}} \times D_{k}(k \geq 2)$. Then the corresponding partition $\lambda(J)$ is $\left(i_{1}+1, i_{1}+\right.$ $\left.1, \ldots, i_{t}+1, i_{t}+1,2 k-1,1^{s}\right)$ where $2 \sum_{j=1}^{t}\left(i_{j}+1\right)+2 k-1+s=2 n$. Thus if $J$ has a component of type $D_{k}(k \geq 2), s$ is odd and $p=2 t+1+s$, while if $J$ has no component of type $D_{k}$ then $s$ is even and $p=2 t+s$. In the former case $n-|J|=(p-2) / 2$, while in the latter, we have $n-|J|=p / 2$. Thus

(7.27) for a parabolic subsystem of type $J$ in $D_{n}$, suppose the corresponding partition $\lambda(J)$ has $p$ parts of which $s$ are equal to 1 ; then

$$
\left\langle\operatorname{Ind}_{W_{J}}^{W}(1), \rho\right\rangle= \begin{cases}(p-2) / 2 & \text { if } J \text { has a component of type } D(s \text { odd }), \\ p / 2 & \text { if } J \text { has no component of type } D(s \text { even), }\end{cases}
$$


(ii)

$$
\left\langle\operatorname{Ind}_{W_{J}}^{W}(1), \xi\right\rangle=(p-2) / 2 .
$$

The equation (7.27)(ii) follows, for example, from (i) by putting $q=1$ in (7.11) and subtracting (use (4.9)).

In the case under consideration (that is, we exclude the case $n$ odd and less than $p),(7.27)$ is sufficient to determine $\left\langle Q_{A}, \rho\right\rangle$ for $s$ even. We have

(7.28) Suppose $J$ is a parabolic subsystem of $D_{n}$ with corresponding partition $\lambda(J)$ having $p$ parts of which $s$ are equal to 1 . Exclude the case where $n$ is odd and less then $p$. Then for a parabolic nilpotent $A$ of type $J$, we have

(i) $\left\langle Q_{A}, \xi\right\rangle=q^{2}+q^{4}+\cdots+q^{p-2}$.

(ii) if $s$ is odd (that is, $J$ has a component of type $D$ ) then

$$
\left\langle Q_{A}, \rho\right\rangle=q+q^{3}+\cdots+q^{p-3} .
$$

(iii) if $s$ is even (that is, $J$ has no component of type $D$ ) then

$$
\left\langle Q_{A}, \rho\right\rangle=q+q^{3}+\cdots+q^{p-3}+q^{p-s / 2-1} .
$$

Moreover the following is an easy consequence of (7.27)(ii) and (7.25).

(7.29) Using the notation of (7.28), suppose that $n$ is odd and less than $p$. Then for odd $s$ (that is, when $J$ has a component of type $D$ ), (i) and (ii) remain valid.

Comparing with (2.9)(iii), this completes the proof of Theorem (2.4).

Note that the case excluded above is where $J$ is a subsystem of type $A_{i_{1}} \times$ $\cdots \times A_{i_{t}}$ of $D_{n}$ where $n$ is odd and $n<2 t+s$ (where $s=2 n-2 \sum\left(i_{j}+1\right)$ ). This is precisely the case $(*)$ in the statement of (2.4).

The non-parabolic case for $G$ of type $B_{n}$ (any $n$ ) or $D_{n}$ ( $n$ even).

We maintain the above notation and discuss the two cases simultaneously, since the notation in Cases 1 and 2 above are consistent with each other. In view of (7.12), (7.13), (7.24) and (7.25) the determination of $\left\langle Q_{A}, \rho\right\rangle$ and $\left\langle Q_{A}, \xi\right\rangle$ for all nilpotent $A \in \mathscr{G}$ reduces to the determination of the $\rho$ - and $\xi$-isotopic components of $H^{2 r+s-2}\left(\mathscr{B}_{A}\right) \quad\left(=H^{p+r-2}\left(\mathscr{B}_{A}\right)\right)$ when $s$ is even. The four equations just mentioned show that if $s>0, H^{p+r-2}\left(\mathscr{B}_{A}\right)_{p, \xi}=2 \rho$ or $2 \xi$ or $\rho+\xi$ (where $M_{\rho, \xi}$ denotes the sum of the $\rho$ - and $\xi$-isotopic components of the module $M$ ), while if $s=0, H^{2 r-2}\left(\mathscr{B}_{A}\right)_{\rho, \xi}=\rho$ or $\xi$.

For the rest of this section write $G=O(2 n+1)$ or $O(2 n)$, according to the case being considered, and write $G^{\prime}=S O(2 n+1)$ or $S O(2 n)$ correspondingly. Define

$$
C_{G}(A)=Z_{G}(A) / Z_{G}(A)^{0} \quad \text { and } \quad C_{G}(A)^{\prime}=Z_{G^{\prime}}(A) / Z_{G^{\prime}}(A)^{0} .
$$


Then $C_{G}(A)^{\prime}$ has index 2 in $C_{G}(A)$ and the structure of $C_{G}(A)$ is well known (cf. Shoji $[18, \S 1]$ ). If the partition $\lambda$ corresponding to $A$ is written $\lambda=\left(1^{n_{1}}, 2^{n_{2}}, \ldots\right)$ (so that $n_{1}=s$ ) then $C_{G}(A)$ is an elementary abelian 2-group with involutory generators $\left\{\alpha_{i} \mid i\right.$ is odd and $\left.n_{i} \neq 0\right\}$.

If $s>0$, write $\theta$ for the (complex) character of $C_{G}(A)$ given by $\theta\left(\alpha_{1}\right)=$ $-1, \theta\left(\alpha_{i}\right)=1,(i \neq 1)$. We also regard $\theta$ as a character of $C_{G}(A)^{\prime}$ by restriction.

Now $Z_{G}(A)$ acts naturally on $\mathscr{B}_{A}$ and $\mathscr{P}_{A}$, whence there is an induced action of $C_{G}(A)$ on $H^{*}\left(\mathscr{B}_{A}\right)$ and $H^{*}\left(\mathscr{P}_{A}\right)$, which turns out to be commutative with the $W$-action. Since the natural map $\mathscr{B}_{A} \rightarrow \mathscr{P}_{A}$ is $Z_{G}(A)$-equivariant, the induced map $H^{*}\left(\mathscr{P}_{A}\right) \rightarrow H^{*}\left(\mathscr{B}_{A}\right)$ is $C_{G}(A)$-equivariant. Moreover the isomorphism in (3.7) is $C_{G}(A)$-equivariant.

(7.30) LemMa. Let $i=r+s / 2-1$.

(i) If $s>0, H^{2 i}\left(\mathscr{P}_{A}\right)=1+\theta$ as $C_{G}(A)$-module.

(ii) If $s=0, H^{2 i}\left(\mathscr{P}_{A}\right)=1$ as $C_{G}(A)$-module.

Proof. Suppose $s>0$. By (7.9) and (7.23), the natural map $H^{2 i}(\mathscr{P}) \rightarrow$ $H^{2 i}\left(\mathscr{P}_{A}\right)$ induced by the inclusion $\mathscr{P}_{A} \hookrightarrow \mathscr{P}$ has a non-trivial image for $i=r+\frac{s}{2}-1$. Since this map is $C_{G}(A)$-equivariant and $C_{G}(A)$ acts trivially on $H^{2 i}(\mathscr{P})$, it follows that $H^{2 i}\left(\mathscr{P}_{A}\right)$ contains a copy of the trivial representation of $C_{G}(A)$.

Now identify $\mathscr{P}_{A}$ as $\mathscr{V}(r, s)$ as above, with notation for the coordinates as in (5.6). Using the explicit description of the action of $Z_{G}(A)$ on $\mathscr{P}_{A}$ given in [18], we see that $\alpha_{j}(j \neq 1)$ acts on $\mathscr{P}_{A}$ via $\alpha_{j}\left(u_{k}\right)=-u_{k}$ (some $k$ ) while $\alpha_{1}\left(z_{1}\right)=-z_{1}$ (the $\alpha_{j}$ fix all other coordinates). Using the filtration $\mathscr{V}(r, s)-\mathscr{V}(r, s-1) \simeq \mathbb{A}^{r} \times \mathscr{X}_{s-1}$ as before, one sees easily that $\alpha_{j}(j \neq 1)$ acts trivially on $H^{2 i}\left(\mathscr{P}_{A}\right)$. It remains only to show that $\alpha_{1}$ acts non-trivially on $H^{2 i}\left(\mathscr{P}_{A}\right)$. In view of the above $\left(C_{G}(A)\right.$-stable $)$ filtration, it is enough to show that $\alpha_{1}$ acts non-trivially on $H_{c}^{s-2}\left(\mathscr{X}_{s-1}\right)$. Changing coordinates, $\mathscr{X}_{k}$ may be written as $\mathscr{X}_{k}=\left\{\left(z_{1}, \ldots, z_{k}\right) \in \mathbb{A}^{t} \mid z_{1}^{2}+\cdots+z_{k-1} z_{k}=1\right\}$ with $\alpha_{1}$-action $z_{1} \mapsto-z_{1}$. We show that $\alpha_{1}$ acts non-trivially on $H_{c}^{k-1}\left(\mathscr{Z}_{k}\right)$ for any $k \geq 1$. For this, observe that $z_{k}=0$ defines a subvariety of $\mathscr{Z}_{k}$ which is isomorphic to $\mathscr{X}_{k-2} \times \mathbb{A}^{1}$ and $\mathscr{X}_{k}-\left(\mathscr{X}_{k-2} \times \mathbb{A}^{1}\right) \simeq \mathbb{A}^{k-1}-\mathbb{A}^{k-2}$. Using this filtration repeatedly, one reduces to the case $k=1$ or $k=2$, where an easy verification finishes the proof. The case $s=0$ is similar and easy.

To deduce $\left\langle Q_{A}, \rho\right\rangle$ from this, we need a property of "Ennola duality of Green functions" (cf. $[18, \S 4])$. The following formula, which is a simplified version, is due to Spaltenstein [24]. 
(7.31) Proposition (Spaltenstein [24]). Let $W$ be a Weyl group containing -1 and let $\chi$ be an irreducible character of $W$. Define the parity $p(\chi)$ by

$$
p(\chi)= \begin{cases}0 & \text { if } \chi(-1)=\chi(1), \\ 1 & \text { if } \chi(-1)=-\chi(1) .\end{cases}
$$

For any $\phi \in\left(C_{G}(A)\right)^{-}$write $\chi_{A, \phi}$ for the character of $W$ corresponding to $(A, \phi)$ under the Springer correspondence. Then

$$
H^{2 i}\left(\mathscr{B}_{A}\right)_{\chi \otimes \phi} \neq 0 \Rightarrow i \equiv d_{A}+p(\chi)+p\left(\chi_{A, \phi}\right) \quad(\bmod 2)
$$

(where $d_{A}=\operatorname{dim} \mathscr{B}_{A}$ ).

Note that the hypotheses apply to our case, type $B_{n}$ (any $\left.n\right)$, type $D_{n}(n$ even).

In our case it is easily verified that $p(\rho)=1$ and $p(\xi)=0$.

(7.32) Lemma. Let $\theta \in C_{G}(A)^{\wedge}$ be as above. Then

$$
p\left(\chi_{A, \theta}\right)= \begin{cases}p\left(\chi_{A, 1}\right) & \text { if } s / 2 \text { is even, } \\ p\left(\chi_{A, 1}\right)+1 & \text { if } s / 2 \text { is odd } .\end{cases}
$$

This follows from the explicit description of the Springer correspondence given in $[18, \S 1]$.

(7.33) Proposition. Maintaining the above notation, let $i=r+s / 2-1$. If $M_{\rho, \xi}$ denotes the sum of the $\rho$-and $\xi$-isotypic components of the $W$-module $M$, we have

(i) if $s / 2$ is odd, $H^{2 i}\left(\mathscr{B}_{A}\right)_{\rho, \xi}=\rho+\xi$, if $s / 2$ is even, $\neq 0$ then $H^{2 i}\left(\mathscr{B}_{A}\right)_{\rho, \xi}$

$$
= \begin{cases}2 \xi & \text { if } G \text { is of type } B_{n}, \\ 2 \rho & \text { if } G \text { is of type } D_{n}(n \text { even }),\end{cases}
$$

(iii) if $s=0$ then $H^{2 i}\left(\mathscr{B}_{A}\right)_{\rho, \xi}= \begin{cases}\xi & \text { if } G \text { is of type } B_{n}, \\ \rho & \text { if } G \text { is of type } D_{n} \text { (n even). }\end{cases}$

Proof. (We assume (7.30), (7.31) and (7.32).) Suppose first $s>0$. From (7.30) we have

$$
H^{2 i}\left(\mathscr{B}_{A}\right)_{\rho, \xi}=1 \otimes \chi_{1}+\theta \otimes \chi_{2}
$$

as $C_{G}(A) \times W$-module, with $\chi_{1}, \chi_{2} \in\{\rho, \xi\}$. Suppose first that $s / 2$ is odd; then $p\left(\chi_{A, \theta}\right) \neq p\left(\chi_{A, 1}\right)$ by (7.32). Hence by (7.31) $\chi_{1} \neq \chi_{2}$, whence (i) follows. If $s / 2$ is even, then $p\left(\chi_{A_{1}}\right)=p\left(\chi_{A_{A}}\right)$ by (7.32) and again 
by (7.31), $\chi_{1}=\chi_{2}$. Write $\chi=\chi_{1}=\chi_{2}$. By (7.9) and (7.31), the map $H^{2 j}(\mathscr{P}) \rightarrow H^{2 j}\left(\mathscr{P}_{A}\right)$ is surjective for $j \neq i=r+s / 2-1$. In particular, if $C_{G}(A)$ acts trivially on $H^{2 j}\left(\mathscr{B}_{A}\right)_{\chi} \neq 0$ for some $j \neq i$, we have $j \equiv i$ $(\bmod 2)$ by $(7.31)$. But for $j \neq i$, we know $H^{2 j}\left(\mathscr{B}_{A}\right)_{\rho, \xi}$ by (7.12), (7.13) and (7.24), (7.25); moreover $r$ is even when $G$ is of type $D_{n}$ and odd when $G$ is of type $B_{n}$. The result (ii) follows.

Note that only case (i) occurs for $A$ of parabolic type. The proof of (iii) is the same as that of (ii).

We summarize the results above in terms of the polynomials $\left\langle Q_{A}, \rho\right\rangle$ and $\left\langle Q_{A}, \xi\right\rangle$.

(7.34) Theorem. Suppose $G$ is of type $B_{n}$ or $D_{n}$ (with $n$ even in the latter case). Let $A$ be a nilpotent element of $\mathscr{G}$ with corresponding partition $\lambda$, which has $p$ parts of which $s$ have cardinality one. Write $r=p-s$. Then, with $\rho, \xi \in \widehat{W}$ as defined above, we have the following.

(i) If $s$ is odd

$$
\left.\begin{array}{l}
\left\langle Q_{A}, \rho\right\rangle=q+q^{3}+\cdots+q^{p-2} \\
\left\langle Q_{A}, \xi\right\rangle=q^{2}+q^{4}+\cdots+q^{p-3} \\
\left\langle Q_{A}, \rho\right\rangle=q+q^{3}+\cdots+q^{p-3} \\
\left\langle Q_{A}, \xi\right\rangle=q^{2}+q^{4}+\cdots+q^{p-2}
\end{array}\right\} \quad \text { if } G \text { is of type } B_{n},
$$

(ii) If $s$ is even

$$
\left.\begin{array}{l}
\left\langle Q_{A}, \rho\right\rangle=q+q^{3}+\cdots+q^{p-2} \\
\left\langle Q_{A}, \xi\right\rangle=q^{2}+q^{4}+\cdots+q^{p-3}+q^{r+s / 2-1} \\
\left\langle Q_{A}, \rho\right\rangle=q+q^{3}+\cdots+q^{p-3}+q^{r+s / 2-1} \\
\left\langle Q_{A}, \xi\right\rangle=q^{2}+q^{4}+\cdots+q^{p-2}
\end{array}\right\} \quad \text { if } G \text { is of type } B_{n},
$$

The proof is a straightforward translation of the results of (7.33) using (7.12), (7.13), (7.24) and (7.25). It seems noteworthy that in spite of the detailed information concerning the $W$-structure of $H^{*}\left(\mathscr{B}_{A}\right)$ which is used to prove (7.34), the formulae for $\left\langle Q_{A}, \rho\right\rangle$ and $\left\langle Q_{A}, \xi\right\rangle$ in the case $s$ even are uniform.

\section{Complements and concluding remarks}

Besides the main theorem (2.4) which describes the occurrence of the reflection representation of $W$ in the "Springer representations" of parabolic type in terms of the cohomology of complex hyperplane complements, our methods have produced the $\left\langle Q_{A}, \rho\right\rangle$ for arbitrary nilpotent $A$ for all cases 
except $S O(4 n+2)$. In addition, whenever $\left\langle Q_{A}, \rho\right\rangle$ is known, so is $\left\langle Q_{A}, \xi\right\rangle$ where $\xi$ is the other non-trivial component of $\operatorname{Ind}_{W(L)}^{W}(1)$ (see (6.6) for $\mathrm{Sp}(2 n),(7.34)$ for $S O(2 n+1)$ and $S O(4 n),(7.28)$ and (7.29) for $S O(4 n+2)$ and $A$ of parabolic type.)

The basic thrust of this work is that the polynomials $\left\langle Q_{A}, R\right\rangle$ should have a description in terms of the geometry of the representation $R$ of $W$.

(8.1) Problem. Find such a description.

It is possible that work such as that of Tanisaki [28] and De Concini, Procesi and Kraft $[6,11]$ on the representations of $W$ on the scheme theoretic intersection of a Cartan subalgebra of $\mathscr{G}$ with a nilpotent orbit might provide such a link.

Consider the special case of (8.1) where $R=\rho_{i}$ the $i$ th "compound" (that is, exterior power) of the reflection representation. In the special case $A=0$, the method of Solomon [21] may be applied to show that

$$
\left\langle\sum_{i=0}^{\operatorname{dim} \mathscr{B}} H^{2 i}(\mathscr{B}, \mathbb{C}) q^{i}, \rho_{j}\right\rangle=\left\langle Q_{0}, \rho_{j}\right\rangle=\sigma_{j}\left(q^{m_{1}}, \ldots, q^{m_{l}}\right)
$$

where $\sigma_{j}$ is the $j$ th elementary symmetric function.

In view of (8.2) one might conjecture as follows.

(8.3) Conjecture. For $A$ of parabolic type $J$, we have

$$
\left\langle Q_{A}, \rho_{j}\right\rangle=\sigma_{j}\left(q^{b_{1}\left(X_{J}\right)}, q^{b_{2}\left(X_{J}\right)}, \ldots\right)
$$

where the notation is as in (2.4).

We know (8.3) is true for $A=0$, for $j=l$ (in which case $\rho_{j}=\varepsilon$, the sign representation), for $j=1$ (the present work) and for certain cases in type $A$.

Here also, in addition to a verification of the conjecture, a geometric explanation would be desirable.

\section{Note added in proof}

We now have an argument which eliminates the exception in Theorem 2.4, and therefore completes the computation of $\left\langle Q_{A}, \rho\right\rangle$ for $A$ of parabolic type. The argument uses a transverse slice to the orbit of $A$ in $\mathscr{G}$. Spaltenstein, in a recent preprint ("On the reflection representation in Springer's theory"), has refined and simplified our method to obtain $\left\langle Q_{A}, \rho\right\rangle$ for any nilpotent $A$ when $G$ is classical. 


\section{References}

[1] D. Alvis and G. Lusztig, 'On Springer's correspondence for simple groups of type $E_{n}$ $(n=6,7,8)$, Math. Proc. Cambridge Philos. Soc. 92 (1982), 65-78.

[2] W. Borho and R. MacPherson, 'Représentations des groupes de Weyl et homologie d'intersection pour les variētés nilpotentes,' C. R. Acad. Sci. Paris A 292 (1981), 707710.

[3] W. Borho and R. MacPherson, 'Partial resolutions of nilpotent varieties,' Astérisque 101-102 (1983), 23-74.

[4] E. Brieskorn, Sur les groupes de tresses (d'après V. I. Arnold), Sém. Bourbaki 1971/72, Lecture Notes in Math., vol. 317, (Springer-Verlag, Berlin, 1973).

[5] P. Deligne and G. Lusztig, 'Representations of reductive groups over finite fields,' Ann. of Math. 103 (1976), 103-161.

[6] C. De Concini and C. Procesi, 'Symmetric functions, conjugacy classes and the flag variety,' Invent. Math. 64 (1981), 203-219.

[7] I. Fary, 'Cohomologie des variétés algébriques,' Ann. of Math. 65 (1957), 21-61.

[8] R. Godement, Topologie algébrique et théorie des faisceaux, Hermann, Paris, 1964.

[9] R. Hartshorne, Residues and duality, Lecture Notes in Math., vol. 20, (Springer-Verlag, 1966).

[10] D. Kazhdan, 'Proof of Springer's hypothesis,' Israel J. Math. 28 (1977), 272-286.

[11] H. Kraft, 'Conjugacy classes and Weyl group representations,' Proc. of the Conférence Internationale, Torún, Pologne, 1980, Astérisque 87-88 (1981), 195-205.

[12] G. I. Lehrer, 'On the Poincaré series associated with Coxeter group actions on complements of hyperplanes,' J. London Math. Soc. (2) 36 (1987), 275-294.

[13] G. Lusztig, 'Green polynomials and singularities of nilpotent classes,' Adv. in Math. 42 (1981), 169-178.

[14] G. Lusztig, 'Characters of reductive groups over a finite field,' Ann. of Math. Studies 107 (Princeton Univ. Press, 1984).

[15] J. S. Milne, Étale cohomology, Princeton Univ. Press (Princeton, N.J., 1980).

[16] P. Orlik and $\mathbf{L}$. Solomon, 'Combinatorics and the topology of complements of hyperplanes,' Invent. Math. 56 (1980), 167-189.

[17] P. Orlik and L. Solomon, 'Coxeter arrangements,' in Proc. Sympos. Pure Math. 40 (1983), 167-189.

[18] T. Shoji, 'On the Green polynomials of classical groups,' Invent. Math. 74 (1983), 239267.

[19] T. Shoji, 'Geometry of orbits and Springer correspondence,' to appear in Proc. Special Period on Unipotent Orbits etc., Astérisque 1988.

[20] L. Solomon, 'Invariants of finite reflection groups,' Nagoya Math J. 22 (1963), 57-64.

[21] N. Spaltenstein, Classes unipotents et sous groupes de Borel, Lecture Notes in Math., vol. 942 (Springer-Verlag, 1982).

[22] N. Spaltenstein, 'On the generalized Springer correspondence for exceptional groups,' in Algebraic groups and related topics, Adv. Studies in Pure Math. 6, pp. 317-338, (Kinokuniya and North-Holland, 1985).

[23] N. Spaltenstein, Problems in algebraic groups, Report of Katata Conference, p. 19 (Osaka 1983).

[24] N. Spaltenstein, 'On Springer representations of Weyl groups containing -1, Arch. Math. (Basel) 44 (1985), 26-28.

[25] T. A. Springer, 'Trigonometric sums, Green functions of finite groups and representations of Weyl groups,' Invent. Math. 36 (1976), 173-207.

[26] T. A. Springer, 'A construction of representations of Weyl groups,' Invent. Math. 44 (1978), 2979-293. 
[27] T. A. Springer and R. Steinberg, 'Conjugacy classes,' Seminar on algebraic groups and related finite groups, Lecture Notes in Math., vol. 131, pp. 167-226 (Springer, 1970).

[28] T. Tanisaki, 'Defining ideals of the closures of the conjugacy classes and representations of the Weyl groups,' Tôhoku Math. J. 34 (1982), 575-587.

University of Sydney

Sydney, NSW 2006

Australia
Science University of Tokyo

Noda Chiba 278

Tokyo

Japan 